\title{
MedienPädagogik
}

Zeitschrift für Theorie und Praxis der Medienbildung www.medienpaed.com

Themenheft Nr. 31: ‘Digitale Bildung〉

Medienbezogene Bildungskonzepte für die «nächste Gesellschaft).

Hrsg. v. Jasmin Bastian, Tobias Feldhoff, Marius Harring und Klaus Rummler.

\section{[Me]nsch - Werkzeug - [I]nteraktion \\ Theoretisch-konzeptionelle Analysen zur «Digitalen Bildung» und zur Bedeutung der Medienpädagogik in der nächsten Gesellschaft}

Thomas Knaus

\section{Zusammenfassung}

Mit Blick auf aktuelle technische und gesellschaftliche Entwicklungen sowie die historischen Wurzeln der Medienpädagogik wird in diesem Beitrag eine Metapher vorgeschlagen, die die Rolle der Disziplin in der nächsten Gesellschaft (Baecker 2007) beschreibt - einer Gesellschaft, die durch digitaltechnikinduzierte Entgrenzungen und Neu-Vernetzungen beeinflusst ist.

Den Ausgangspunkt theoretisch-konzeptioneller Überlegungen bildet das Sozialisationskonzept, aus dem die Medienpädagogik bereits Anleihen machte. Die gegenwärtigen Entwicklungen um die Spezifika des Digitalen werfen jedoch neue Fragen auf, die eine ReVision des Konzepts nahelegen: Zentral wird dabei die Frage sein, welchen Anteil Medien in einer «digitalen» und «vernetzen» Welt am «Prozess der dynamischen und produktiven Verarbeitung von Realität» (Hurrelmann 2006, 28) haben. Auslöser von Konstruktionsprozessen sind nicht nur andere Menschen, sondern auch deren mediale Artefakte - Dinge, die Individuen kommunikativ vernetzen und die zunehmend mittels digitaler Werkzeuge erstellt, gestaltet und manipuliert werden. Hierdurch beeinflusst das medienschaffende Subjekt die Prozesse der Realitätsverarbeitung und -konstruktion seines sozialen Umfeldes - zumal nicht nur das Medium selbst gestaltbar ist, sondern auch die digitalen Werkzeuge. Aufgrund ihrer Programmierbarkeit, Adaptivität und Konvergenz werden sie selbst zu Kommunikationsmedien. Besondere Aufmerksamkeit gilt also der Kommunikation zwischen Menschen sowie der Kommunikation zwischen Menschen und Dingen - der symbolischen Mensch-Maschine-Interaktion.

Diese Erkenntnisse liefern Einsichten über das Lernen mit und über Medien, die einer Weiterentwicklung der schulischen Medienpraxis mit dem Ziel der Förderung «Digitaler Bildung» als Grundlage dienen. Sie verweisen zugleich auf das Desiderat einer zuständigkeitsklärenden interdisziplinären Vernetzung der Medienpädagogik mit technisch-gestalterischen Disziplinen wie der Informatik. 
Human - [Me]dia - [I]nteraction. Theoretical and Conceptual Considerations on «Digital Literacy» in Schools and the Role of Media Education in the Next Society

\begin{abstract}
In the context of current technological and social developments and with a view to the historical roots of Media Education, this contribution presents a metaphor which describes the role of Media Education in the Next Society (Baecker 2007) - a society in which digital technology blurs traditional boundaries in education and science and opens up new opportunities for «digital» socialization.

The starting point for theoretical and conceptual considerations is provided by a General Educational approach - the theory of socialization - from which Media Education has already borrowed in the past. However, technological developments have given rise to new issues which suggest that this approach should now be revisited. At the forefront of this reappraisal lies the role the media will occupy in a «digital» and «networked» world and what part it will play in the "model of dynamic and productive processing of reality" (Hurrelmann 2006, 28). Construction processes are not only triggered by other people, but also by medial artefacts - things that provide a communicative link between people and which are being increasingly generated, designed and manipulated using digital technology. Individual media creators use these tools to influence the way in which they process reality and construct their social environment; they are able to this largely because it is not just the medium itself but - due to the programmability, adaptivity and the convergence of digital tools - the tools themselves which are also being generated, thereby rendering them a medium of communication in their own right. Particular attention should therefore be paid to the communication between people as well as between people and things - to the symbolic human-machine interaction.

This study provides insights into the ways in which people learn with and about media, findings which serve to develop educational media use and support «Digital Literacy». These findings also reveal the need to establish a link between Media Education and technical design-related disciplines such as Informatics which would also help to demarcate the boundaries of responsibility between the fields.
\end{abstract}


Form: «Digitale Bildung» - Vom Hashtag zum Konzept

Der Hashtag «Digitale Bildung» wird aktuell häufig gebraucht, jedoch selten hinterfragt. Der Gehalt des Begriffs steht und fällt wohl mit seiner «intuitiven» Rezeption: Digitalen Medien, wie Smartphones und Tablets, wird eine intuitive Bedienbarkeit unterstellt. Sie wird technisch über die Antizipation typischer Nutzungsszenarien realisiert: Die Software «kennt» die üblichen Anforderungen und Ziele der Nutzenden und navigiert sie entsprechend dieser üblichen Pfade. Die Technik bestimmt den Weg und wir nehmen ihn - aus Bequemlichkeit. So wird der Intuition gefolgt, anstatt Entscheidungen durch den diskursiven Gebrauch des Verstandes zu treffen. Wird digital, als «omnipräsentes Adjektiv» (Knaus 2016b, 100), aus ähnlichen Gründen so unkritisch und unreflektiert in nahezu alle gesellschaftlichen, kulturellen, ökonomischen und politischen Vollzüge übernommen?

Zur Kritik am omnipräsenten Adjektiv wird hier eine alternative fachliche Sichtweise vorgeschlagen: Wenn Informatikerinnen und Informatiker das Adjektiv digital hören, dann denken sie zunächst an etwas «Binäres» - an Einsen und Nullen. In einer binären Welt lässt sich aus einem Schaltzustand heraus alles konstruieren. Irgendwie scheint diese Komplexitätsreduktion jedoch nicht zu dem zu passen, was wir mit «digitalen Welten», «digitaler Gesellschaft» oder auch «digitaler Bildung» ausdrücken wollen. Digitalisierung bezeichnet üblicherweise die Übertragung analoger Erscheinungsformen in diskrete Werte mit dem Ziel, diese elektronisch zu verarbeiten - u.a. zu speichern. Eine «analoge Gesellschaft» lässt sich aber nicht einfach in eine digitale Gesellschaft transformieren. Ist also der Begriff der Digitalisierung zutreffend, wenn er auf eine Gesellschaft angewendet wird - auf Kultur, auf Bildungsfragen, auf Schule oder auf das menschliche Lernen? Präziser müssten die Fragen doch lauten: Wie verändern sich unser Leben, Zusammenleben und unsere Umwelt in einer technologisch und technisch induzierten gesellschaftlichen Umbruchsituation - und welche Fähigkeiten werden für die persönliche Entwicklung, die Integration und ein souveränes Miteinander benötigt? «Wie wirken digitale Medien auf Individuen und die Gesellschaft, wie kann man Informationen beurteilen, eigene Standpunkte entwickeln und Einfluss auf gesellschaftliche und technologische Entwicklungen nehmen? Wie können Gesellschaft und Individuen digitale Kultur und Kultivierung mitgestalten?» (Brinda et al. 2016). Zugegeben, «digitales Something» spricht sich schneller und prägt sich auch besser ein. Doch trifft beispielsweise «Digitale Bildung» wirklich den Kern dessen, was wir damit meinen? Wer ist beispielsweise Expertin oder Experte in Sachen «Digitaler Bildung»? Und wer wäre zuständig? Präzisere Analysen erscheinen notwendig. 
Die Analyse soll ihren Anfang in diesem Beitrag ${ }^{1}$ mit einem knappen Blick in die Ideengeschichte der Medienpädagogik finden und daraufhin Thesen zur Bedeutung digitaler Medien, Werkzeuge und Werkstoffe für die Konstitution von Subjekt und Gesellschaft entfalten.

\section{Turn: Vom Medienschutz zur Medienpädagogik}

Um die 1920er und 1930er Jahre erschöpfte sich der pädagogische Umgang mit Medien primär darin, Kinder und Jugendliche «vor Schund- und Schmutzliteratur, aber auch vor negativen Einflüssen aus Filmen [zu] schützen» (Seel/Hanke 2015, 897). So wurde beispielsweise 1926 das Gesetz zur Bewahrung der Jugend vor Schund- und Schmutzschriften erlassen. Nach einer sehr dunklen Zeit, die durch Zensur und Propaganda geprägt war, entstanden in den Nachkriegsjahren die Freiwillige Selbstkontrolle der Filmwirtschaft - FSK, das Gesetz zum Schutze der Jugend in der Öffentlichkeit - JÖSchG, das Gesetz über die Verbreitung jugendgefährdender Schriften - GjS sowie die entsprechende Bundesprüfstelle - BPjS (vgl. Schorb 1995; Vollbrecht 2001, 25-48; Süss et al. 2013, 62-63 und 78) - die historische Funktion erster medienpädagogischer Ansätze liesse sich daher mit der Rolle einer «Torwächterin» treffend umschreiben.

Zeitgleich etablierten sich Bestrebungen, Jugendliche über Filmgespräche zu einer kritischen Haltung gegenüber Film- und Medieninhalten zu erziehen (vgl. Vollbrecht 2001, 25-48; Süss et al. 2013, 66 ff.). Hierin können erste Tendenzen einer Medienpädagogik erkannt werden, die nicht nur verbietend-schützend agiert, sondern die das Individuum stärken und bilden möchte - und ihm hierüber nachhaltigen Schutz zukommen lässt. Der Einfluss der Kritischen Theorie der Frankfurter Schule in den 1960 und 1970er Jahren verstärkte die Bestrebungen, alle Menschen zu kritischen Bürgerinnen und Bürgern erziehen zu wollen. Dieser Zeitpunkt in der Geschichte markiert eine wesentliche Umbruchsituation: den Turn des Menschenbildes in der Pädagogik vom passiven Rezipienten hin zum aktiven Subjekt. Dies war eine Veränderung, die nicht zuletzt auch durch die Entwicklung neuer technischer und medialer Potentiale befördert wurde (vgl. Schorb 1995, 47) und die den Boden für aufklärende, alltags- und handlungsorientierte sowie partizipatorische Ansätze in der Medienpädagogik ebnete (vgl. Baacke 1996, 46-50; Süss et al. 2013, 69-70). Damit etablierte sich eine «begleitende statt bewahrende Pädagogik» (Hoffmann 2008, 49).

1 Den Beitrag haben (in alphabetischer Reihenfolge) Valentin Dander, Olga Engel, Carolin Hahn, Nastasja Müller, Gerhard Tulodziecki und zwei anonyme Reviewerinnen/Reviewer mit wertvollen Kommentaren versehen. Für die hilfreichen Anregungen und Korrekturen, Diskussionen und die kritische Kommentierung dieses Textes danke ich Euch und Ihnen sehr! 
Mit den aktuellen gesellschaftlichen Entwicklungen um die so genannte «Digitalisierung» (vgl. Kapitel «Form: «Digitale Bildung» - Vom Hashtag zum Konzept») scheint sich nun jedoch die bewahrpädagogisch geprägte Medien-Historie zu wiederholen: Seit einigen Jahren beteiligen sich Kriminologen (Pfeiffer 2003) sowie Psychiater (Spitzer 2005/2012) am medienpädagogischen Diskurs. Unter anderem hierdurch drängen Politik und öffentliche Meinung Medienpädagoginnen und Medienpädagogen erneut in schützend-bewahrende Positionen. Dabei sollten wir uns doch eigentlich inzwischen daran gewöhnt haben, dass noch jede mediale Innovation gesellschaftliche Ängste vor deren negativen Folgen schürte (vgl. u.a. Deterding 2011, 103-105). Natürlich sollten diejenigen nachhaltig geschützt werden, die sich (noch) nicht selbst schützen können. Eine ausschliesslich schützende Position aber - genauso wie das blosse Antrainieren von Fertigkeiten und Fähigkeiten - ignoriert, worauf es in Anbetracht einer rasanten Technik- und Gesellschaftsentwicklung ankommt: Medienkompetenz. In der Community ist dies Common Sense. So schrieb beispielsweise Heinz Moser in seiner seit 1995 mehrfach aufgelegten Einführung in die Medienpädagogik: Mir «[...] erscheint ein rein bewahrpädagogischer Ansatz im Zeitalter einer Medien- und Informationsgesellschaft kaum mehr ein realistisches und wünschenswertes Ziel» (Moser 2010a, 143) - die Rolle der Torwächterin passt demnach nicht (mehr) zur Medienpädagogik einer nächsten Gesellschaft, die nach Dirk Baecker eine "Computergesellschaft» sein wird (Baecker 2007; vgl. auch Drucker 2002 zu «next society»). Doch diese Erkenntnis dringt bedauerlicherweise nur schwer in die öffentliche Diskussion vor. Dabei ist eines unstrittig: In unseren Gesellschaften ist digitale Technik bereits omnipräsent und der Weg zurück ist nicht möglich - soweit jedenfalls, um es ganz soziologisch mit Georg Jellinek zu sagen, die «normative Kraft des Faktischen» (Jellinek 1914, 338).

\section{Thesen zur Bedeutung des Digitalen in der nächsten Gesellschaft}

Die folgenden drei Thesen sollen den Boden für eine Re-Vision eines klassischen handlungstheoretischen Konzepts bereiten, das unter der Berücksichtigung derzeitiger technologischer und technischer Entwicklungen aktueller denn je erscheinen wird.

\section{Digitale Medien beeinflussen unsere Umweltwahrnehmung (1. These)}

Das ist Nikki. Nikki ist Jazz-Sängerin. Nikki sagte vor kurzem im ZEITmagazin, dass sie als Zwölfjährige aus lauter Neugier «Jazz» gegooglelt habe und daraufhin eine bekannte Jazz-Sängerin wurde (vgl. ZEITmagazin, 31. Juli 2014). 


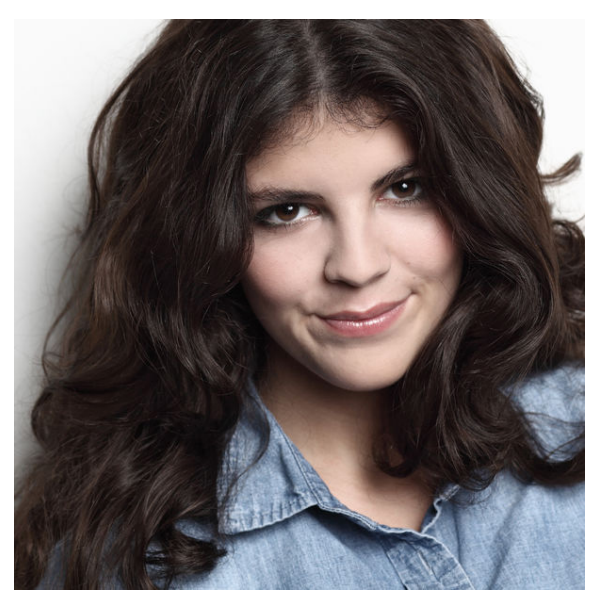

Abb. 1.: Nikki Yanofsky, Nikki Yanofsky @ Steven Haberland (http://static.universal-music.de/ asset_new/237006/881/view/Nikki-Yanofsky----Steven-Haberland.jpg).

Hieran erstaunt eigentlich... nichts. Mit diesem Beispiel - das auch ein ganz anderes hätte sein können - soll die Relevanz digitaler Medien für unsere Weltwahrnehmung unterstrichen werden. Dieses Beispiel überzeugt möglicherweise noch deutlicher, wenn ich verrate, dass Nikki aus Kanada stammt und in Montreal aufgewachsen ist das ist just die Stadt, in der jährlich das weltweit grösste Jazz-Festival stattfindet: Um mit Jazz in Berührung zu kommen, hätte Nikki also auch einfach vor die Tür gehen können. So kann konstatiert werden: Digitale Medien beeinflussen in hohem Masse unsere Wahrnehmung und entsprechend die produktive Verarbeitung von Wirklichkeit.

\section{Digitale Technik ist gesellschaftsprägend und omnipräsent (2. These)}

Dass Technik Gesellschaften verändert - und schon immer veränderte - ist bekannt: Der historische Technikeinsatz war im Wesentlichen durch die Erleichterung physischer Arbeit gekennzeichnet: So revolutionierte beispielsweise die Eisenbahn die Mobilität; im Bausektor machte der Technikeinsatz das kräftezehrende Handwerk nicht nur sicherer und für Menschen erträglicher, sondern ermöglichte auch neue Bauwerksarten. Technik hatte auch direkten Einfluss auf das soziale Miteinander: «Die Einführung der Sprache konstituierte die Stammesgesellschaft, die Einführung der Schrift die antike Hochkultur, die Einführung des Buchdrucks die moderne Gesellschaft und die Einführung des Computers die nächste Gesellschaft» (Baecker 2007, 7). Maschinen revolutionierten also die physische Arbeit und die Medientechnik, wie die Schrift und der Buchdruck sowie der Computer, reformiere die geistige Arbeit (vgl. u.a. Nake 1992; Schelhowe 1997 und 2016, 44) und die Verarbeitung von Sinn (vgl. Meder 1998; Drucker 2002; Swertz 2006; Baecker 2007). Als jeweils 
dominierende Kommunikationsmedien prägten sie das gesellschaftliche Miteinander. Doch was änderte sich mit der rasanten Verbreitung des mobilen und vernetzten Computers?

Kann ein technisches Gerät unsere Gesellschaften derart verändern, dass wir nach einer medialen und strukturellen Re-form eine «nächste Gesellschaft» und damit eine Kulturreform ausrufen? Sind die technikinduzierten Brüche tatsächlich so wesentlich? In jedem Fall geht es nicht um ein Gerät: Die Mensch-Computer-Relation veränderte sich in den letzten Jahren bereits entscheidend. So arbeiteten vor wenigen Jahren mehrere Personen an einem Computer - in Banken waren Menschen an Terminals tätig; in Universitäten konnten Forschende Rechenkapazitäten buchen. In den letzten beiden Jahrzehnten dominierte der «persönliche» Computer - der PC und damit die Eins-zu-eins-Relation von Mensch und Maschine. Heute genügt vielen von uns nicht mehr nur ein Gerät. Damit änderte sich das Verhältnis von Mensch und Maschine erneut. Mit der steigenden Zahl digitaler Geräte, die einen Menschen umgeben, sind aber nicht nur die sichtbaren Geräte, wie Smartphone, Tablet, Notebook und PC gemeint, sondern auch weniger auffällige Computer in Alltagsgegenständen, wie Motorsteuerungen und Bremsassistenten im Auto, Haus- und Heizungssteuerungen oder auch «smarte» Kühlschränke und Zahnbürsten. In nicht allzu ferner Zukunft wird beinahe jeder Gegenstand des täglichen Lebens einen Computer enthalten und mit den anderen Gegenständen vernetzt sein, was als IOT - Internet of Things - bezeichnet wird und die Omnipräsenz digitaler Werkzeuge zementiert.

Es geht aber in Anbetracht digitaler Technik nicht nur um Quantitäten und technische Geräte, sondern um eine neue Qualität von Technik, eine veränderte soziale Funktion von Technik und damit auch um ein neues Verständnis der Mensch-Maschine-Interaktion.

\section{Digitale Werkzeuge machen Realität (3. These)}

An der Benutzerschnittstelle, am Interface - also an der Stelle, an der Technik zum «Gegenüber» und damit zum Medium wird - ist es zunächst irrelevant, ob es sich um ein analoges oder digitales Medium handelt, denn die mittels analoger Technik hergestellten Bilder, Filme oder Tondokumente stehen bezüglich ihrer medialen Erscheinung digital produzierten Dokumenten in nichts nach (vgl. Manovich 2001, 48; Knaus 2009, 19-44). Während das Medium primär die äussere Erscheinungsform, als Objekt der Wahrnehmung die Schnittstelle zum Menschen darstellt, umfasst der Werkzeugbegriff auch die zugrundeliegende technische Basis des digitalen Geräts, die aufgrund ihrer Programmierbarkeit, Adaptivität, Konvergenz und technischen Vernetzung umfassende Gestaltungsmöglichkeiten der medialen Erscheinungsformen sowie der eigenen Funktionalitäten bietet und damit über Potentiale verfügt, die über jene der analogen Medien herausreichen (vgl. u.a. Knaus 2015; Knaus 2016a/b; Knaus 2017a). 
Weiterführender als die Differenzierung analoger und digitaler Technik ist also vielmehr die Unterscheidung der Technik in Bezug auf ihre soziale Funktion (vgl. Knaus/ Engel 2015; Knaus 2018): Erscheint die Technik in konkreten gesellschaftlichen Vollzügen primär als Medium, als Werkstoff oder als Werkzeug? Gerade bezüglich digitaler Technik mag diese Unterscheidung zunächst künstlich oder gar antiquiert wirken zumal sie nicht selten verschwimmt und Menschen doch von Beginn an ihre dingliche Umwelt gestalteten: So berichtete das Journal Nature kürzlich, dass die bisher ältesten Werkzeugfunde auf 3,4 Mio. Jahren datiert werden konnten (vgl. Nature, Vol. 466, 857-860). Die Unterscheidung zwischen Medium, Werkstoff und Werkzeug ist aber gerade unter Berücksichtigung der zuvor benannten Spezifika des Digitalen wesentlich und weiterführend, da sie verdeutlicht, dass das Medium nicht nur dann beteiligt ist, wenn wir Umwelt wahrnehmen, wie zuvor beispielhaft mit der Jazzsängerin Nikki erläutert (weitere Beispiele vgl. Moser 2010a, 18 ff.), sondern auch dann, wenn wir unsere Umwelt sowie digitale Werkstoffe mittels digitaler Werkzeuge erschaffen oder gestalten: Codes und Software werden zu Werkstoffen, aus denen sich Werkzeuge herstellen und kombinieren lassen; Werkzeuge, mit denen wiederum digitale Werkstoffe und Medien produziert und manipuliert werden können.

Die individuelle Produktion und aktive Gestaltung analoger (Massen)Medien war zwar bereits in «analogen Zeiten» grundsätzlich möglich, aber stets aufwändig - man denke nur an die historische Vervielfältigung von Texten oder die ersten Filme aus den Anfängen der Kinematografie. Erst mit dem Aufkommen der Digitaltechnik konnten auch diejenigen, die üblicherweise rezipieren, das Objekt der Wahrnehmung direkt beeinflussen - es manipulieren, wie es der Informatiker Reinhard Keil nennt: "Zum ersten Mal in unserer Mediengeschichte kann das Objekt der Wahrnehmung auch unmittelbar zum Objekt der Manipulation werden» (Keil 2006, 67). Damit konnte das digitale Medium nicht nur rezipiert werden, sondern auch ohne grössere Aufwände selbst produziert und «interaktiv» manipuliert werden.

Hierbei ist wesentlich, dass der alltagssprachlich eher negativ besetzte Begriff der Manipulation nicht falsch verstanden wird: Es ist hier nicht der aus dem Französischen stammenden Fachbegriff aus der Soziologie oder Psychologie gemeint - es geht also nicht um gezielte oder verdeckte Einflussnahme oder gar um Konditionierung. Und es geht auch nicht darum, dass Medien Menschen «manipulieren» würden. Der Begriff der Manipulation sollte im Folgenden vielmehr in seinem eigentlichen Wortsinn verstanden werden: So ist manus das lateinische Wort für «Hand» und plere bedeutet «füllen». Man könnte also wörtlich übersetzen: «eine Handvoll haben» oder etwas passender: «die Dinge in der Hand haben». Damit ist gemeint, dass wir digitale Medien nicht nur rezipieren, sondern auf das Objekt der Wahrnehmung auch selbst handelnd zugreifen können. 


\section{Re-Vision: Kommunikation der Dinge}

Zusammenfassend kann in Anbetracht der drei vorgestellten Thesen festgehalten werden, dass digitale Medien in hohem Masse unsere Wahrnehmung von (Um-)Welt und die produktive Verarbeitung von Wirklichkeit beeinflussen. Aufgrund der Omnipräsenz digitaler Medien und technischer Netze sind auch unsere sozialen Netzwerke ständig verfügbar. Da wir zunehmend auch mittels digitaler Medien Realität erzeugen, kann Realitätskonstruktion in unmittelbarer Weise erlebt und es können in der Revision digitaler Artefakte Reflexionsprozesse angestossen werden. Damit sind digitale Medien und Soziale Netze nicht nur ständig beteiligt, wenn wir andere und uns selbst wahrnehmen und inszenieren (vgl. Kapitel «Thesen zur Bedeutung des Digitalen in der nächsten Gesellschaft»), sondern aufgrund der Speicherbarkeit und Vernetzung digitaler Artefakte stehen mediale Konstruktionen zeitlich und örtlich ungebunden (vgl. Kapitel «De-Form: Entgrenzungs- und Vernetzungspotentiale des Digitalen») zur persönlichkeits- und gesellschaftsbildenden Reflexion zur Verfügung. Prozesse von Sozialisation werden demnach zunehmend medial vermittelt und medial konstruiert: Die Wahrnehmung von innen und aussen, die Konstruktion von Umwelt und die Subjekt- und Gesellschaftsbildung werden von digitalen Medien und Werkzeugen - die wohlgemerkt omnipräsent sind - durchsetzt und beeinflusst (vgl. u.a. Sutter 1999; Carstensen et al. 2014).

Dies vorausgesetzt, verwundert es, dass in den Sozialisationstheorien digitale Medien, Werkstoffe und Werkzeuge noch immer eine so untergeordnete Rolle spielen und bisher in der Sozialisationsforschung vorrangig noch als Massenmedien wahrgenommen werden: In der eingängigeren Beschäftigung mit Sozialisationstheorien - beispielsweise der programmatischen Schrift von Dieter Geulen und Klaus Hurrelmann (1980) - fällt auf, dass Medien darin überhaupt keine Rolle spielen; in späteren Neuauflagen von Klaus Hurrelmann zur Sozialisationstheorie kommen zumindest «die Massenmedien» in Form von knappen Absätzen zur «Sozialisationswirkung von Massenmedien» und «der alltägliche Umgang mit Massenmedien» zur Geltung (Hurrelmann 2006, 27 und 254 ff.). Im umfassenden Handbuch Sozialisationsforschung von Klaus Hurrelmann, Matthias Grundmann und Sabine Walper (2008), wird nahezu jeder relevante Aspekt der Sozialisation beleuchtet, aber die Bedeutung der Medien vom Psychologen Helmut Lukesch unter dem Titel «Sozialisation durch Massenmedien» bearbeitet; in der aktuellen Neuauflage des Handbuchs wird die «Sozialisation in der mediatisierten Gesellschaft» aus soziologischer Sichtweise thematisiert (vgl. Lange 2015). In den kommunikationswissenschaftlichen oder medienpädagogischen Arbeiten zur «Mediensozialisation» (vgl. u.a. Baacke 1996, 38-43; Fritz et al. 2003; Hoffmann/Mikos 2010; Vollbrecht/Wegener 2010; Hoffmann et al. 2017) werden, zu diesem Schluss kommt Dieter Spanhel, «die Medien in ihrer Bedeutung für den Sozialisationsprozess isoliert oder unter eingeschränkten Perspektiven konzeptualisiert» (Spanhel 2013, 30). So werden Medien beispielsweise als Orte der Sozialisation 
bezeichnet und damit nur sehr spezifische Funktionen der Medien für die Persönlichkeitsentwicklung betont. Gerade in Arbeiten zur Mediensozialisation herrscht jedoch inzwischen Einigkeit darüber, dass sich das Verständnis von Medien als Sozialisationsinstanz - also neben Familie und Schule - überholt hat, da Medien inzwischen in allen Instanzen vorhanden und prägend sind (vgl. Kapitel «Thesen zur Bedeutung des Digitalen in der nächsten Gesellschaft»).

Warum aber ist es problematisch, wenn auch die omnipräsenten, digitalen Medien, Werkstoffe und Werkzeuge als «Sonderfälle» und Massenmedien verstanden werden? Diese Interpretation unterschätzt zunächst die sozialisatorische Relevanz von Medien und reduziert diese darüber hinaus auf ihre Rezeption. Damit ignoriert dieses Verständnis das Gestaltungspotential, das gerade digitale Medien und soziale Netze dem Individuum zur Verfügung stellen (vgl. Kapitel «Thesen zur Bedeutung des Digitalen in der nächsten Gesellschaft» und «De-Form: Entgrenzungs- und Vernetzungspotentiale des Digitalen») und verweist auf ein überholtes Menschenbild (vgl. Kapitel «Turn: Vom Medienschutz zur Medienpädagogik»). Damit wird nicht nur das Potential digitaler Medien als «Instrumente im Prozess der Sozialisation» (Schorb 2005, 386) unterschätzt, sondern auch ihre förderliche Eignung mittels Symbolen und Beziehungsreflexionen strukturelle Kopplungen zwischen physischen und sozialen Systemen herzustellen (vgl. Schmidt 1998; Sutter 1999; Spanhel 2013, 32; Thye 2013) und damit selbst zum Objekt von Sozialisation zu werden (vgl. Deterding 2011, 120f.).

Nach den für die deutschsprachige Sozialisationsforschung prägenden Autoren, Dieter Geulen und Klaus Hurrelmann, entsteht und entwickelt sich die Persönlichkeit eines Menschen «in wechselseitiger Abhängigkeit von der gesellschaftlich vermittelten sozialen und materiellen Umwelt» (Geulen/Hurrelmann 1980). Damit wird als Sozialisation die Summe aller Lernprozesse bezeichnet, die der Mensch in seiner Entwicklung zu einer handlungsfähigen Persönlichkeit durchläuft. Wenn nun also, wie zuvor konstatiert, das Leben zwar «analog» ist, Kommunikation und Kollaboration aber zunehmend «digitalisiert» wurden und entsprechend medial stattfinden (vgl. u.a. Knaus 2016c), dann kann die Persönlichkeitskonstitution und -entwicklung hiervon nicht unbeeindruckt bleiben (vgl. u.a. Carstensen et al. 2014, 13; Knaus 2018; Tulodziecki 2018).

Nach dem Symbolischen Interaktionismus (vgl. Blumer 1969; Mead 1973) - einer Theorie, die für die handlungs- und rollentheoretische Perspektive als wesentliche soziologische Basistheorie innerhalb des Sozialisationskonzepts, prägend war (vgl. Hurrelmann 2006, 91-96; Tillmann 2010, 170-198) - ist Kommunikation zwischen Individuen der Schlüssel zur Subjektkonstitution: «Kommunikation dient [demnach] nicht nur dem Transport von Botschaften, sondern primär der Konstitution und Entwicklung von Subjekt und Gesellschaft» (Schachtner/Duller 2014, 85). Charles Horton Cooley, einer der geistigen Väter von George Herbert Mead, nimmt an 
- möglicherweise inspiriert durch Johann Wolfgang von Goethe -, dass die Impulse für die Persönlichkeitsentwicklung vom Anderen ausgehen: «Der Mensch erkennt sich nur im Menschen» (Goethe 1999, 611-697: Antonio, II, 3). Für Cooley geschieht dieses Erkennen mittels Kommunikation, er schreibt dazu: «lt is through communication that we get our higher development. The faces and conversation of our associates; books, letters, travel, arts [...] supply the stimulus and framework for all our growth» (Cooley 1972, 63). George Herbert Mead entwickelte in Anlehnung an Cooley ein Modell, das die Konstitution des Subjekts durch Kommunikation konzeptionalisiert. Das Subjekt konstituiert sich demnach immer «innerhalb des gesellschaftlichen Erfahrungs- und Tätigkeitsprozesses» (Mead 1973, 177). Von seinem Schüler Herbert Blumer wurde dieses Modell dann weiter ausgebaut. Darin entsteht die Persönlichkeit - von Mead und Blumer als [Self] bezeichnet - als Produkt zweier Grössen: Dem «sozialen Selbst», dem [Me], und der psychischen Komponente des [I].

Das $[\mathrm{Me}]$ steht für die in einer Gesellschaft existierenden gemeinsamen Normen, Regeln, Werthaltungen, also: für die sozialen Konsense (vgl. Mead 1973, 198). Es präsentiert die Vorstellung dessen, wie die anderen Menschen - also: das soziale Umfeld - ein Individuum sehen und wie es sich nach der Interpretation ihrer Erwartungen zu verhalten habe. Das $[\mathrm{Me}]$ wiederum «speichert» die intersubjektiv ausgehandelten Erwartungen und stellt darüber hinaus handlungsleitende Strukturen und Orientierungen zur Verfügung. Das [I] vertritt - gegenüber dem [Me] - impulsive und spontane Energien der Person, die zwar durch das [Me] «gezügelt» werden, aber doch eine unabhängige Grösse der Persönlichkeit darstellen. Durch ebendieses Zusammenwirken von [I] und [Me] bildet sich das [Self], also: das Selbst - das Selbstverständnis. Mead und Blumer gehen davon aus, dass Individuum und Gesellschaft in sich prozesshaft verwoben sind - für sie sind Individuum und Gesellschaft zwei aufeinander bezogene und vernetzte Dimensionen, die erst im Wechselspiel die Entstehung des menschlichen Subjekts ermöglichen (vgl. auch Joas 1991; Tillmann 2010, 16-20). Dieses komplexe Zusammenwirken in «Spiel» und «Wettkampf» - [Play] und [Game] - muss man sich nach dem Kommunikationswissenschaftler Friedrich Krotz als kommunikativen Prozess vorstellen (vgl. Krotz 1998, 72), der nach aussen wahrnehmbar, sich aber durchaus auch als «innerer Dialog» abspielen kann. Selbstbewusst, identitätsbewusst sein, heisst dann im Grunde: dank der gesellschaftlichen Beziehungen zu anderen für seine eigene Identität zum Objekt zu werden. Das ist Aufgabe des [Mind] - am besten wohl mit «Geist» zu übersetzen -, der als die reflexive Intelligenz des Menschen verstanden wird.

Cooley, Mead und Blumer sind sich darin einig, dass diese «sozialisierende» Kommunikation nicht nur zwischenmenschlich erfolgt, sondern auch zwischen Mensch und Dingen möglich ist - eine Erkenntnis übrigens, die in den letzten Jahren in 
Vergessenheit ${ }^{2}$ geriet (vgl. Baacke 1996, 55; Meyer-Drawe 1999; Nohl 2011, 8). Kommunikation findet also auch dann statt, wenn Dinge uns ein Handeln nahe- oder auferlegen. Ein Beispiel hierfür ist der Berliner Schlüssel: Der Berliner Schlüssel (den man auch als Doppelschlüssel oder Durchsteckschlüssel kennt) ist ein Schlüssel mit zwei identischen Bärten. Er wird nach dem Aufschliessen durch das Schloss hindurch geschoben und ermöglicht daraufhin das Zuschliessen der Durchgangstür von der anderen Seite. Dieser Schlüssel ist ein treffendes Beispiel für Dinge, die Menschen ein bestimmtes Handeln auferlegen - man könnte auch sagen: Dinge, die «kommunizieren» (vgl. Latour 1993; Knaus 2009). Zu Dingen, die «kommunizieren», gäbe es zahlreiche Beispiele aus der Kunst oder Architektur - so wird Ihre Stimmung, während Sie diese Zeilen lesen, möglicherweise zu einem nicht unwesentlichen Teil von der Architektur des Raumes abhängig sein, in dem Sie sich gerade befinden.

Auch digitale Medien, Werkstoffe und Werkzeuge «kommunizieren» und können so unser Handeln beeinflussen (vgl. Meder 1998; Swertz 2006, 73). In Bezug auf die Massenmedien hat der kanadische Philosoph Marshall McLuhan mit der vielzitierten Erkenntnis the Medium is the Message darauf hingewiesen, dass Medien nicht neutral und demnach niemals nur «Behältnisse» für Informationen sind (McLuhan 1968). Dass wir heute im Gegensatz zum Buch, Film, Fernsehen oder der Architektur unmittelbaren und gestalterisch-kreativen Einfluss auf das digitale Medium selbst nehmen können, indem wir seine technische Grundlage aufgrund ihrer Programmierbarkeit und softwarebasierten Adaptivität gezielt manipulieren können, konnte er seinerzeit bestenfalls erahnen.

\section{De-Form: Entgrenzungs- und Vernetzungspotentiale des Digitalen}

Im Rahmen meiner Recherchen zu den Spezifika des Digitalen in und für Bildungskontexte entstanden Arbeiten um Begriffe wie Interaktion, Störung, Widerstand, Adaptivität, Manipulation und Vernetzung, die jeweils für mich nicht nur technische Dimensionen sind, sondern als solche Auswirkungen auf die soziale Funktion von Technik haben - und damit für allgemeinpädagogische und insbesondere auch medienpädagogische Fragen sehr anknüpfbare Perspektiven darstellen.

Für die weiteren Ausführungen greife ich die Aspekte Entgrenzung und daraufhin ermöglichte Neu-Vernetzungen heraus und werde an drei Beispielen aktuelle Entwicklungen darstellen, die auch Potentiale für Bildungs-, Lehr- und Lernkontexte bieten. Im weiteren Verlauf stelle ich eine Studie vor, die zeigt, dass diese Potentiale

2 Arndt Nohl greift diese Gedanken vier Jahrzehnte später auf und konstatiert: «Bildung, Sozialisation und [...] auch Lernen und Erziehung [können] nicht nur in Interaktion mit anderen Menschen, sondern auch im Austausch mit Dingen vonstattengehen [...]. Doch diese Materialität pädagogischer Prozesse ist in der Pädagogik so an den Rand ge(d)rückt [...]. Wenn wir Erziehungswissenschaftler/innen von «Gegenständen` sprechen, dann meinen wir zumeist Themen und Diskurse, die unserer geistigen Durchdringung bedürfen» (Nohl 2011, 8; Herv. TK). 
in der schulischen Praxis - zumindest in der Breite - bisher ungenutzt bleiben (vgl. Kapitel «Re-Turn to earth: Medienpraxis in der Schule»), obwohl das Lernen mit und über Medien in der Schule wünschenswert wäre (vgl. Kapitel «Von der Notwendigkeit des Lernens mit und über Medien»). Daraufhin werden vier Desiderate entfaltet, deren gemeinsamer Nenner die Überwindung tradierter Grenzen ist (vgl. Kapitel «Desiderata: Crossing Boundaries»).

\section{Real oder Virtuell}

Als um die Jahrtausendwende Boris Becker mit dem Spruch «Bin ich schon drin?» Werbung für den amerikanischen Onlineanbieter AOL machte, mag die Unterscheidung zwischen «real» und «virtuell» noch hilfreich gewesen sein, aber ist sie es heute auch noch, wenn wir «smart \& mobil» (Friedrich et al. 2015) und always on sind? Für Kinder und Jugendliche verwischen nach aktueller Studienlage (vgl. u.a. Calmbach et al. 2016; DIVSI 2014; MPFS 2014; MPFS 2016) die Grenzen zwischen online und offline: Die beiden Welten - ob analog-real oder digital-virtuell - gehören zusammen, da die Grenze zusehends verschwimmt und unsere Realitäten sich ausdehnen - sie expandieren. Diese Expansion wird sichtbar sowie praktisch und didaktisch nutzbar mittels digitaler Medien, die die physische Realität mittels computergenerierter Zusatzinformationen vermengen (Mixed Reality) oder erweiterten (Augmented Reality): Praktisch wird bei der computergestützten Erweiterung der Realität ein von einer Kamera bereitgestelltes Live-Bild mittels zusätzlicher (computergenerierter) visueller Informationen ergänzt, wie wir es zum Beispiel von Navigationssoftware kennen. Gerade beim Selbstorganisierten Lernen oder für Stillarbeitsphasen können erweiterte Realitäten in Form von didaktischen Hilfestellungen gute Dienste leisten. ${ }^{3}$

\section{Raum und Zeit}

Eine weitere Entgrenzung: Es geht um nichts Geringeres als die Erweiterung von Raum und Zeit. Bereits eines der ersten technischen Medien - die Schriftsprache löste zeitliche Dimensionen auf: Auch wenn der Geschichtenerzähler irgendwann nicht mehr unter uns war, lebten seine Geschichten in Büchern oder auch Bildern weiter. Soziale Eingebundenheit war bisher an räumliche Nähe gebunden, während wir uns durch das Netz - und nicht zuletzt mittels «Sozialer Netze» - heute kontextbezogen und interessengeleitet neu vernetzen können. Aufgrund der Konvergenz physischer und digitaler Wirklichkeiten können wir also Grenzen überwinden und damit neue Formen der Vergesellschaftung erleben (vgl. u.a. Wenger 1998; Jenkins 2006; Knaus 2009; Knaus 2016a).

3 Bei Kolleginnen und Kollegen am IMIS in Lübeck konnte ich miterleben, wie Augmented Reality im Kunst-, Geschichts- und Deutschunterricht eingesetzt wird, so beispielsweise für eine Führung durch die Lübecker Altstadt zum Leben und literarischen Wirken von Günther Grass. 


\section{Kommunikations- und Handlungsspielräume}

Wie bereits ausgeführt, lösen digitale Medien die Reduktion der Medien auf Wahrnehmungsprozesse zugunsten manipulierbarer - auch hier wieder im eigentlichen Wortsinne, nämlich: gestalt- und handhabbarer - Zugänge auf. Damit können digitale Medien das Verständnis vom «realitätsverarbeitenden Subjekt» (Hurrelmann 2006) zum «realitätskonstruierenden Subjekt» (Beer 2007) auch ganz praktisch unterstützen. In der praktischen und lebensweltorientierten Medienarbeit wird damit erfahrbar, wie wir unsere (Um-)Welten gestalten.

Mit der Vorstellung, dass Kommunikation nicht nur zwischen Menschen erfolgt (vgl. Kapitel «Re-Vision: Kommunikation der Dinge»), erweitern wir unsere Kommunikationsräume und mit der digitalen Expansion des Mediums - vom Objekt der Wahrnehmung hin zum Werkzeug (vgl. Kapitel «Thesen zur Bedeutung des Digitalen in der nächsten Gesellschaft») - auch unsere Handlungsspielräume. Es ist davon auszugehen, dass Konstruktionen des lebensweltlichen Umfelds und damit auch (trans- und cross-)mediale Produktionen aus der kreativen Medienarbeit in Kultureller Bildung und Schule interne Konstruktionsprozesse befördern (vgl. u.a. Tulodziecki et al. 2010, 187-198; Knaus 2018), vor allem dann, wenn eine aktive Reflexion der Symbole, Situationen und Beziehungen stattfindet (vgl. Kapitel «Re-Vision: Kommunikation der Dinge»).

Soweit zu den - zumindest potentiellen - Entgrenzungen und Neu-Vernetzungen; der Blick in die aktuelle Medienpraxis an deutschen Schulen «erdet» leider recht rabiat.

\section{Re-Turn to Earth: Medienpraxis in der Schule}

All das wäre heute schon in Schule und Unterricht möglich: die kreative Vernetzung inner- und ausserhalb der Schule (sowohl zeitlich als auch räumlich), die Erweiterung der Realitäten in trans- und cross-mediale Formen - sofern sie didaktisch hilfreich sind -, aktivierender Unterricht mit Medien sowie projektbezogene oder fächerintegrierte kreative Medienarbeit, die eigene Gestaltungs- und Handlungsspielräume erfahrbar macht und zur persönlichkeitsbildenden Reflexion anregen kann (vgl. Knaus 2016c; Knaus 2018).

\section{Rezeption oder Aktion?}

Auf der Suche nach Unterricht, der die Potentiale des Digitalen nutzt, hospitierte ich im Unterricht in fast 200 Schulen. Die Erkenntnisse meiner Untersuchungen lassen sich generell nicht kurz zusammenfassen, aber in Bezug auf eine immer wieder bestätigte Erfahrung gelingt die knappe übergreifende Analyse: In digitalen Medien steckt bekanntermassen das Potential zur Aktivierung. Wenn jedoch heute digitale 
Medien im schulischen Unterricht eingesetzt werden, dann wird in der Regel mit ihnen präsentiert, im Internet bzw. WWW recherchiert oder in PDFs gelesen. Werden die gesammelten Materialien nur auf diesen einen Aspekt hin untersucht - nämlich, ob Unterricht mit digitalen Medien eher passiv-rezeptiv oder aktiv-konstruierend ist dann zeigt sich, dass in der Schule bisher im Wesentlichen rezipiert wird.

Werden in Studien «schulische ICT» (Lorenz/Bos 2015, 23f.), die «Digitale Schule und vernetztes Lernen» (Bitkom 2015) oder allgemein die schulische Mediennutzung in den Blick genommen und Lehrende nach quantitativen Umfängen und Frequenzen eines Unterrichts, der «die Anwendung von Computern thematisiert» (Lorenz/Bos 2015, 23) befragt, werden die zuvor genannten Elemente üblicherweise als Bestandteile «digitalen Unterrichts» eingeordnet. Ich halte dies insofern für irreführend, als dass Studien, die blosse Häufigkeiten der Techniknutzung im Unterricht oder auch quantifizierbare Ausstattungs- und Zugangsdaten erheben, oft digital mit «gut» gleichsetzen. Natürlich können das Präsentieren, Recherchieren und Lesen Elemente eines sehr guten Unterrichts sein, aber die Potentiale digitaler Medien könnten meines Erachtens und in Anbetracht der im vorherigen Kapitel exemplarisch entfalteten Möglichkeiten besser genutzt werden.

\section{Substitution oder Redefinition?}

Das SAMR-Modell von Ruben Puentedura (vgl. Abbildung 2) eignet sich gut, um zu differenzieren, ob eine technische Innovation (1) lediglich darin besteht, ein Medium oder Werkzeug in eine andere Form zu übertragen ohne dessen Funktionalität zu verändern (Substitution); ob (2) tradierte Medien und Werkzeuge funktional erweitert werden (Augmentation); ob Technik (3) dazu beiträgt, Aufgaben neu zu gestalten (Modification); oder ob (4) auch neuartige Einsatzszenarien und Möglichkeiten entstehen, die zuvor undenkbar waren (Redefinition). 

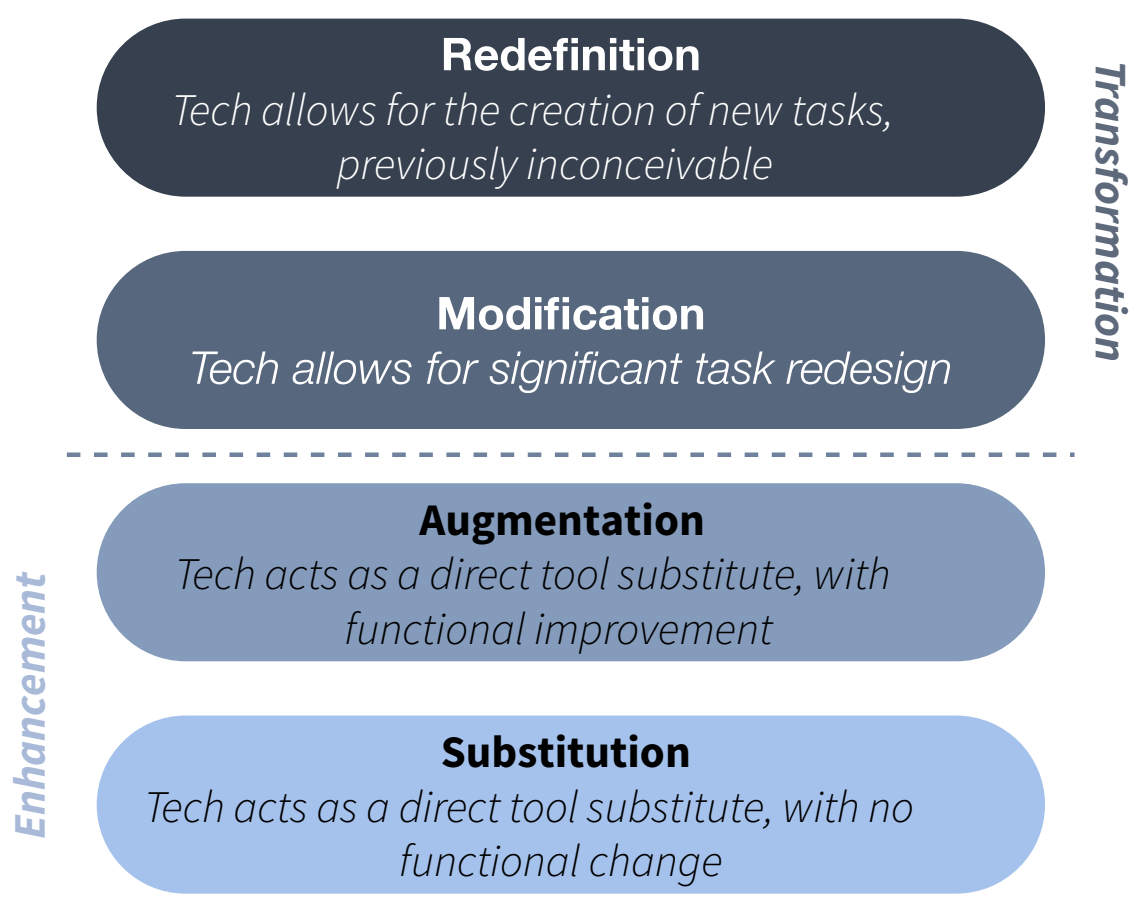

Abb. 2.: SAMR-Model von Ruben Puentedura (vgl. www.hippasus.com/rrpweblog/).

Wird die jeweilige Mediennutzung der untersuchten Schulen in das SAMR-Modell eingeordnet, zeigt sich, dass in den wenigsten Schulen der Unterricht über das Implementierungsstadium der Substitution, also das Ersetzen analoger Medien und Werkzeuge durch ihre digitale Entsprechung, hinausgeht. ${ }^{4}$ Es ist wohl eher eine rhetorische Frage, ob in der Substitution analoger Medien die konzeptionellen Potentiale digitaler Medien und Werkzeuge zum Vorschein kommen können.

Die Antwort auf die Frage, ob mit diesem Unterricht die Medienkompetenz von Schülerinnen und Schülern gefördert werden könnte, fiele vermutlich ähnlich frustrierend aus. Dabei wäre es wichtiger denn je, dass sich die Schule in puncto Medienerziehung und Förderung von Medienkompetenz einmischt.

4 Dabei waren die Voraussetzungen zur Mediennutzung in Schule und Unterricht nie besser: Vor zwei Jahrzehnten, zur Zeit der Initiative "Schulen ans Netz», wettrüsteten die Kommunen und Kreise bezüglich der Schüler-pro-PC-Quote: Finanzkräftigere Kommunen erreichten damals ein Verhältnis von 8 Lernenden pro Computer (vgl. u.a. Engel et al. 2014, 51). Ziel war natürlich eine Vollausstattung, das heisst auf eine Schülerin bzw. einen Schüler sollte ein Computer kommen. Nun ist sie da - die Vollausstattung: in den Taschen der Schülerinnen, Schüler und Studierenden (vgl. Knaus 2013, 34; MPFS 2016, 6, 8 und 23). Bring Your Own Device lautet das Stichwort, aber in den meisten Schulen ist die Nutzung von Smartphones verboten (vgl. auch MPFS 2016, 48). Dass diese (landes- oder schulweiten) «Handyverbote» üblicherweise nicht deren didaktische Nutzung betreffen, wissen leider die wenigsten Lehrenden (aktuelle rechtliche Regelungen der einzelnen Bundesländer finden sich unter: http://www.handysektor.de/paedagogenecke/handyordnung/ handy-in-der-schule-regelungen-derbundeslaender.html). 
Von der Notwendigkeit des Lernens mit und über Medien

In der öffentlichen Diskussion fällt nicht selten das Argument, dass Schülerinnen und Schüler keinen Qualifikationsbedarf im kompetenten und selbstbestimmten Umgang mit digitalen Medien hätten, dass also die Schule ihnen diesbezüglich nichts beibringen könne. Fragt man jedoch Schülerinnen und Schüler, ob ihnen mediale Produktionsprozesse oder die «Etikette» schriftlicher Korrespondenz geläufig sind, sie einschätzen können, wie Big Data Analytics und Algorithmen ihre Weltwahrnehmung und Handeln beeinflussen, sie eine komplexe Formel in einer Tabellenkalkulation nutzen, eine Formatvorlage oder kollaborativ Texte erstellen können, ein souveränes Mail- und Kalendermanagement betreiben oder welche Prüf- und Qualitätskriterien sie bei einer Recherche anlegen, dann würde ersichtlich - wie auch die Ergebnisse der ICILS-Studie (vgl. Bos et al. 2014, 123-141) belegen, dass es noch viel zu lernen gibt. Zur Illustration des Arguments lässt sich auch eine Analogie zum Deutschunterricht herstellen: Wenn Kinder in die Grundschule kommen, sind sie in der Regel der deutschen Sprache bereits mächtig und können beispielsweise Grammatik intuitiv nutzen, wissen aber noch wenig um die dahinterstehende Logik. Bezüglich des schulischen Qualifikationsauftrags ist der Aspekt des Lernens mit Medien als ein Bestandteil der Medienkompetenz demnach ein nach wie vor wesentliches Element. Gäbe es darüber hinaus noch weitere Argumente für das Lernen über Medien?

Studien, wie u.a. die KIM- und JIM-Studien des MPFS, zeigen zwar, dass der ausstattungs- und zugangsbezogene Digital Divide nicht zutrifft (vgl. auch Moser 2010b), das heisst: Fast alle Menschen verfügen inzwischen über persönlichen Zugriff auf digitale und vernetzte Geräte. So sind laut der JIM-Studie 2016 Jugendliche in Deutschland mit Handy bzw. Smartphone, Computer und Internetzugang nahezu vollausgestattet (vgl. MPFS 2016, 6, 8 und 23). Inzwischen wissen wir aber auch, dass die individuelle Mediennutzung von Kindern und Jugendlichen in hohem Masse von der familiären Mediennutzung abhängig ist: Ob beispielsweise Medien in der primären Sozialisationsinstanz - der Familie - nur unterhaltungsbezogen oder auch informationsbezogen genutzt werden, korreliert mit der Mediennutzung und dem Bildungsstand der Eltern (vgl. MPFS 2016, 11f., 28f. und 42f.). Damit verfolgt schulische Medienpädagogik und insbesondere das Lernen über Medien ein weiteres Ziel, nämlich (aller Schwierigkeiten zum Trotz) Chancengleichheit zu befördern. Unterricht mit und über Medien ist demnach nicht nur im Sinne einer Qualifizierung der nächsten Generation sinnvoll, sondern auch ein wichtiges Element zur Verbesserung der Entwicklungschancen aller Schülerinnen und Schüler und damit eine wesentliche Voraussetzung für eine höhere Durchlässigkeit des Bildungssystems. Die sekundäre Sozialisationsinstanz - das Bildungssystem - und hier konkret: die Schule sollte das Lehren und Lernen mit Medien und das Wissen über Medien mit dem Ziel der Verbesserung der Entwicklungschancen aller Menschen in die Curricula aufnehmen. 


\section{Desiderata: Crossing Boundaries}

In Anbetracht der grossen Fragen - beispielsweise der Frage, wie wir künftig leben wollen oder welches Wissen und Können Schülerinnen und Schüler in der nächsten Gesellschaft für ein souveränes Miteinander und gutes Aufwachsen benötigen - offenbart sich Wünschenswertes: Desiderata, deren Gemeinsamkeit im Hinterfragen tradierter Grenzen liegt. Wie die folgenden exemplarischen Ausführungen zeigen, ermöglicht die Infragestellung und Überschreitung dieser Grenzen vielerorts kreative Neu-Vernetzungen.

\section{Schule weiterdenken: Vernetzen, unterstützen und Kooperationen fördern}

Die Medienpädagogik formuliert nicht nur Anforderungen an die Schule, sondern kann gleichermassen auch kreative Zugänge für das Lernen mit und über Medien sowie die informatische Bildung anbieten. Förderlich hierfür wäre die kooperative Vernetzung zwischen der Institution Schule und den Einrichtungen Kultureller Bildung und ausserschulischer Medienarbeit - die Infragestellung der bisher nur selten überwundenen Grenze zwischen formalen und non-formalen Bildungskontexten. Mittels medienpädagogischer Projektarbeit und Aktiver Medienarbeit nach subjekt- und handlungsorientierten Ansätzen in der Schule (vgl. u.a. Schorb 1995; Baacke 1996, 46-50; Tulodziecki 1997) bestünde die Chance, digitale Medien als Verbindungsglied für etwas zu nutzen, das nicht unbedingt getrennt voneinander sein muss: die Lebenswelt der Schülerinnen und Schüler und das (schulische) Lernen. Hiermit würde nicht nur die Rolle der Schule als Lebensraum gestärkt, sondern den Schülerinnen und Schülern würden auch neue anknüpfbare Erfahrungsräume bereitgestellt (vgl. u.a. Niesyto 2004; Wagner/Ring 2016, 147 f.; Brüggen et al. 2017; Knaus 2017b, 40 f.; Knaus 2018). Wie die Ausführungen im Kapitel «De-Form: Entgrenzungs- und Vernetzungspotentiale des Digitalen» zeigen, kann beispielsweise das aktivierende Potential digitaler Medien schon heute in und für den Unterricht genutzt werden; digitale Medien und Werkzeuge erfordern - ermöglichen es mitunter aber auch - bestehende organisatorische Grenzen, wie tradierte Stundentaktungen, Fächergrenzen, Raumkonzepte und Kooperationsverbote, infrage zu stellen.

\section{«Digitalisierung» der Gesellschaft gestalten:}

Medienkompetenz ist weiterhin wichtig, aber nicht genug

Da in der nächsten Gesellschaft (Baecker 2007) nur das «mediengebildete Subjekt» auch ein handlungsfähiges Subjekt (Hurrelmann 2002, 111; Knaus et al. 2017, 2 f.; Tulodziecki 2018) ist, bleibt Medienkompetenz weiterhin relevant. Doch zunehmend liegt es nicht mehr nur in der Hand der oder des Einzelnen, die Kontrolle über die eigenen (personenbezogenen) Daten zu behalten. Eine Person kann sich noch so 
überlegt im WWW und in Sozialen Netzwerken bewegen: Wenn unscheinbare Apps persönliche Daten sammeln - wer liest schon die langen Nutzungsvereinbarungen (die sich mit jedem Update ändern können) - ist es mit der «Datensouveränität» passé: So ist beispielsweise zur Nutzung des Messengers WhatsApp das Freigeben des persönlichen Adressbuchs erforderlich. Durch diese Freigabe werden auch Namen und Kontaktdaten von Personen übertragen, die sich möglicherweise gegen die Nutzung des Dienstes entschieden haben. Personen, die im Adressbuch eines anderen WhatsApp-Kontakts gespeichert sind, können nicht souverän und fallbezogen über die Speicherung und Weitergabe ihrer persönlichen Daten entscheiden (weitere Beispiele siehe Knaus 2018). Es bedarf also ergänzend zur Förderung individueller Medienkompetenz (vgl. Kapitel «Von der Notwendigkeit des Lernens mit und über Medien») und einem konzeptionellen Technikverständnis (vgl. Kapitel «Von der Notwendigkeit einer Technik- und Algorithmuskritik») einen gesellschaftlichen Diskurs darüber, was Technik und global vernetzte Unternehmen dürfen (vgl. Niesyto 2016). Dieser Diskurs muss in Regeln und (Selbst-)Verpflichtungen - oder allgemein: Normen - möglichst transparent und nachvollziehbar kodifiziert werden. Da das Netz und darin operierende Unternehmen Staatsgrenzen überwinden, sollten auch diese Normen idealerweise grenzübergreifend gedacht, diskutiert und vereinbart werden.

\section{Qualifizierung ermöglichen:}

\section{Grundbildung Medien und medienpädagogische Kompetenz}

Die wohl wichtigste Voraussetzung für das Lernen mit und über Medien in der Schule ist die medienpädagogische Kompetenz angehender Lehrerinnen und Lehrer (vgl. Blömeke 2000, 377; Tulodziecki 2012; Niesyto 2012): Lehrende sollten entsprechend nicht nur über eigene Medienkompetenz verfügen, sondern darüber hinaus auch die Fähigkeit besitzen, die Medienkompetenz ihrer Schülerinnen, Schüler und Studierenden zu fördern. Idealerweise sollten alle pädagogischen Fachkräfte die Gelegenheit erhalten, sich medienpädagogisch zu qualifizieren. Vereinzelt findet diese Erkenntnis curricularen Niederschlag in Form einer medienpädagogischen Grundbildung (vgl. u.a. Niesyto 2012), Grundbildung Medien (vgl. u.a. Niesyto 2014, 125 und 128), integrierender Veranstaltungen in der ersten Phase der Lehrendenbildung oder in Lehramt-Erweiterungsstudiengängen (vgl. u.a. Stiller/Bolz 2016, 126 f.); bedauerlicherweise sieht es abseits weniger «Leuchttürme» im deutschsprachigen Raum bezüglich der systematischen Förderung medienpädagogischer Kompetenz und der Grundbildung Medien noch immer recht düster aus.

Wichtig ist daher, dass die Anstrengungen der KMK zu «Medienbildung in der Schule» von 2012 sowie die Anstrengungen in den Ländern zur curricularen Verankerung der Medienbildung unbedingt fortgeführt werden. 
Die genannten bildungspolitischen Entscheidungen münden allesamt in Vorhaben, die auch innerhalb der Medienpädagogik Weiterentwicklungen und Neuvernetzungen erfordern: Exemplarisch können hier der überfällige Professionalisierungsdiskurs (vgl. u.a. Knaus et al. 2017) sowie die nun nötigen Umsetzungen des jüngst entwickelnden Orientierungsrahmens für die Entwicklung von Curricula für medienpädagogische Studiengänge und Studienanteile (vgl. DGfE 2017) genannt werden.

\section{Disziplingrenzen überwinden: Brüche und Offenheit als Chance}

Eine weitere nötige Grenzüberwindung und Neuvernetzung betrifft uns selbst bzw. unser Fach und Berufsfeld: Die Medienpädagogik ist - im Gegensatz zu den sie umgebenden Wissenschaften - eine offene Disziplin. Edwin Keiner bezeichnet sie daher nach Peter Meusburger auch als «fractured-porous discipline» (Keiner 2015, 16; Meusburger 2009, 117), eine Disziplin also, die über eher geringeren Konsens im Hinblick auf Theorien und Methoden verfügt, sich durch hohe Diversität auszeichnet und sich nur schwach gegen Einflüsse anderer Disziplinen abgrenzen kann - man könnte auch sagen: die über eine «negative Handelsbilanz) verfügt. Das hört sich zunächst nicht vorteilhaft an, für mich liegt darin aber eine der Stärken der Medienpädagogik: Denn diese Offenheit ermöglicht, dass die Medienpädagogik Einflüsse aus anderen Disziplinen interessiert aufnimmt und kreativ verarbeitet. Dies entspricht nicht nur einem modernen Wissenschaftsverständnis, sondern diese offene Haltung ergibt sich für eine Sozialwissenschaft auch aus der zuvor beschriebenen technologisch und technisch induzierten gesellschaftlichen Umbruchsituation (vgl. Kapitel «Thesen zur Bedeutung des Digitalen in der nächsten Gesellschaft» und «De-Form: Entgrenzungs- und Vernetzungspotentiale des Digitalen»).

Ein Aspekt, der in der Community nicht gleichermassen geteilt wird, ist die Notwendigkeit der interdisziplinären und überfachlichen Zusammenarbeit mit Forschenden aus beispielsweise der Informatik (vgl. Knaus 2017b). Warum sich die Medienpädagogik aber gerade von den technisch-gestalterischen Disziplinen inspirieren lassen, mitreden und mitgestalten sollte, möchte ich nachfolgend darlegen.

\section{Von der Notwendigkeit einer Technik- und Algorithmuskritik}

Medienkritik ist eine zentrale Dimension der Zielperspektive der Medienpädagogik - der Medienkompetenz. Sie bezieht grundlegende Wahl- und Bewertungsprozesse, das Unterscheiden, Vergleichen, Bewerten von Fakten, Eigenschaften und Qualitäten hinsichtlich der gesellschaftlichen Medienentwicklung und das individuelle Medienhandeln ein (vgl. Baacke 1997; Ganguin 2004; Niesyto 2006; Niesyto 2008). Medienkritik lässt sich nach Sonja Ganguin in mehrere Dimensionen unterscheiden, die aufeinander aufbauen: Wahrnehmungsfähigkeit, Decodierungsfähigkeit, Analysefähigkeit, 
Reflexionsfähigkeit und Urteilsfähigkeit (vgl. Ganguin 2004, 4). So gehört es beispielsweise zur reflektierenden Urteilskraft, die Objektivität des Wahrgenommenen zu beurteilen. Dies ist nicht trivial, da wir beispielsweise Bildern oder Bewegtbildern intuitiv einen Wahrheitsgehalt zusprechen - sie erscheinen uns «objektiv» (vgl. Knaus 2018). Bilder können jedoch manipuliert werden.

Aufgrund der Programmierbarkeit und Adaptivität digitaler Werkzeuge (vgl. Kapitel «Thesen zur Bedeutung des Digitalen in der nächsten Gesellschaft») trifft Manipulierbarkeit gleichwohl nicht nur auf Bilder zu, sondern hält - gemeinsam mit digitaler Technik - in allen Lebensbereichen Einzug: Wer beispielsweise heute zu einer Bank geht und um einen Kredit bittet, wird die affirmative oder negative Antwort nicht der Tatsache verdanken, eine Bankberaterin oder einen Bankberater überzeugt zu haben, sondern vielmehr einem Algorithmus, der die Kreditwürdigkeit der bzw. des Beantragenden «ermittelt». Während noch vor kurzem Menschen über die Kredit- und Vertrauenswürdigkeit von Menschen entschieden, erledigen dies in der nächsten Gesellschaft Maschinen. Zugleich entscheiden Algorithmen nicht nur über die Zuverlässigkeit der Kundschaft, sondern finden überhaupt nur Einsatz, weil man ihre Entscheidungen für zuverlässiger hält als die Urteile von Menschen, welche sich von Emotionen und visuellen Eindrücken (ver)leiten lassen können. Der Technik wird folglich eine Objektivität zugeschrieben, über die menschliche Subjekte nicht verfügen - die interessengeleitete Einflussnahme sollte sich damit reduzieren. Doch sind Algorithmen objektiv?

Aufgrund der «Menschengemachtheit» der kodifizierten Handlungsvorschriften ist die Objektivitätsunterstellung nicht haltbar: Wie Texte und Bilder ist alle Technik - und damit auch Codes und Algorithmen - menschengemacht und damit potentiell fehlerbehaftet. Ausserdem kann sie Interessen Dritter enthalten und damit subjektive Wünsche und Ziele in vermeintlich «objektiven» Entscheidungsprozessen dominieren lassen. Wenn der Computer geistige Arbeit erleichtert (vgl. u.a. Nake 1992; Schelhowe 1997 und 2016, 44; Swertz 2006, 72 f.), ja sogar zunehmend Aufgaben bewältigt, die Menschen kognitiv nicht leisten können, entsteht ausserdem eine $A b$ hängigkeit von Technik bei der Erhebung und Auswertung grosser Datenmengen. Die ersten Erfahrungen mit Big Data Analytics zeigen, dass auf der Grundlage dieser für Menschen unüberschaubaren Datenmengen und komplexen Prozesse zunehmend relevante Entscheidungen getroffen werden (vgl. Gapski 2015; Aßmann et al. 2016), die von Menschen bestenfalls rudimentär nachvollzogen werden können.

Desiderat ist daher eine gesellschaftliche Entmystifizierung von Technik und informatischer Prozesse sowie die Entwicklung einer kritischen Haltung nicht nur Medien - der «technischen Oberfläche», den Interfaces - gegenüber, sondern auch bezüglich Algorithmen, Codes und den von ihnen erzeugten Daten: Werden maschinelle Handlungsanweisungen und Codes akribisch geprüft, wenn die ersten (Test-) Ergebnisse plausibel erscheinen? Wer setzt die Grenz- und Schwellwerte, auf deren 
Grundlage Maschinen «entscheiden» und «lernen»? Welche Auswirkungen für eine Gesellschaft hat es, wenn primär männliche Programmierer Codes generieren? Wie transparent und nachvollziehbar sind Algorithmen - vor allem die, welche personenbezogene Daten verarbeiten und, wie im vorangestellten Beispiel, mitunter sogar generieren? Diese Fragen stellen (und in gesellschaftlichen, politischen und ökonomischen Kontexten diskutieren) zu können, setzt ein grundlegendes Verständnis von Technik voraus. ${ }^{5}$ Aus diesem Grund ist Medienkritik in der nächsten Gesellschaft (Baecker 2007) breiter zu fassen, als es Dieter Baacke (1996), Gerhard Tulodziecki (1997), Stefan Aufenanger (1997) und Heinz Moser (2000) für eine jüngst vergangene Zeit noch definieren konnten - für eine Zeit, in der Mediennutzung durch Freiwilligkeit geprägt und in der die Übernahme von Verantwortung im Sinne einer informationellen Selbstbestimmung seitens des Subjekts noch möglich war (vgl. Knaus 2018).

In einer Gesellschaft, in der fundamentale Entscheidungen von Algorithmen abhängen, «der gedächtnisfähige Computer [...] in der Gesellschaft mitzukommunizieren beginnt, wie man dies bisher nur von Menschen kannte» (Baecker 2007, 9) und zur gesellschaftlichen Partizipation die Mensch-Maschine-Interaktion obligatorisch ist (vgl. Meder 1998; Swertz 2006, 66 und 72 f.), sollte Medienkritik um eine Technikund Algorithmuskritik erweitert werden (vgl. Knaus 2018). Ein Grundverständnis für technische Prozesse wird zur essentiellen Voraussetzung dieser erweiterten Medienkritik. Dies meint nicht, dass jeder Mensch technische Prozesse umfänglich erschliessen oder gar «coden» bzw. «programmieren» können sollte - wie immer wieder überschnelle Forderungen nach diversen spezifischen Fertigkeiten die politischen und curricularen Diskussionen bestimmen (vgl. u.a. KMK 2016), sondern gemeint ist die Fähigkeit zur kritischen Analyse jeglicher medialer und technischer Artefakte sowie die Kompetenz, deren Provenienz und Anspruchsgruppen zu hinterfragen und durchschauen zu können. Medienkompetenz sollte daher und in Anbetracht der neuen sozialen Funktion von digitalen Medien, Werkstoffen und Werkzeugen in der nächsten Gesellschaft um ein Grundverständnis für Technologien, technische Abläufe und Prozesse - beispielsweise im Sinne eines «Computational Thinking» (Wing 2006) - erweitert werden (vgl. Knaus 2016b, 103 f. und 106-116; Knaus 2018).

\section{Re-Form: Medienpädagogik und Informatik}

Medienkompetenz ist ein Begriff, der in seiner traditionellen Auffassung recht wenig mit Technik zu tun hat (vgl. Schelhowe 2007) und im Alltagsverständnis sogar nicht selten ausschliesslich auf technische Fertigkeiten verkürzt wird (vgl. Aufenanger 1997, 3; Knaus 2016b, 107; Knaus 2017a, 63). Dabei ging es doch Dieter Baacke, der

5 Jeanette Wing bezeichnet dieses grundlegende Technikverständnis als Computational Thinking (Wing 2006) und versteht darunter die Fähigkeit, informatische Denk- und Herangehensweisen zu durchschauen. In den kommenden ICILS-Studien (ab 2018) soll diese Fähigkeit als zusätzlicher Kompetenzbereich abgefragt werden. 
den Begriff in Anlehnung an das Kompetenzkonzept von Noam Chomsky sowie die Arbeiten von Karl-Otto Apel und Jürgen Habermas prägte, nicht um die Technik, die Geräte oder die Medien, sondern um Kommunikation und Kooperation (vgl. u.a. Baacke 1973; Baacke 1996, 51-55), also die Fähigkeit eines Menschen, Medien, digitale Werkzeuge und Technik im Allgemeinen souverän für eigene Ziele und Bedürfnisse zu nutzen und selbst-, medien- und gesellschaftsbezogen zu reflektieren, kreativ und partizipativ zu handeln sowie analytisch und strukturell Wissen zu erwerben (vgl. Baacke 1996, 96-100; Knaus 2016b, 109). In diesem Sinne geht es auch heute nicht um die Technik, sondern um die Frage, wie und wozu wir digitale Werkstoffe und Werkzeuge nutzen (wollen). Im Mittelpunkt stehen damit im Wesentlichen noch immer Kommunikation und Kooperation - nur eben vor allem und weiter zunehmend in ihrer medialen Form auf digitaler Basis. Wenn jedoch digitale Technik über die Funktion des Mediums hinausreicht und an der Herstellung von (Medien-)Inhalten beteiligt ist (vgl. Schelhowe 2007, 45 f.) und selbst Interpretationen vornimmt - wie semantische Technologien (vgl. Knaus 2016b, 113) und beispielhaft die «Antwortmaschine» Wolfram|Alpha oder das AddOn Evri-Toolbar -, rückt (wie im vorherigen Kapitel bereits hergeleitet) ein weiteres Ziel von Medienkompetenz in den Fokus der Betrachtung: die Kenntnis der technischen und organisatorischen Bedingungen der Wissens- und Medienproduktion (vgl. u.a. Tulodziecki et al. 2010, 184 f.; Niesyto 2017, 266; Tulodziecki 2018). Es geht jedoch - wie bereits angemerkt - nicht nur um die mediale Erscheinungsform und Prozesse auf Anwendungsebene (die sichtbare Technik, die Interfaces und ihre organisatorischen Bedingungen), sondern auch und gerade in Anbetracht digitaler Technik um die technische Basis, die Software und Handlungsanweisungen - zumal diese die mediale Oberfläche mittels eingeschriebener («programmierter») oder eigenständiger («selbstlernender») Anweisungen steuern.

Wichtig ist also, dass idealerweise alle Menschen über die Kenntnis verfügen, was hinter der Benutzerschnittstelle - hinter dem Interface der Maschine - vorgeht. Eine um ein konzeptionelles Technikverständnis und mindestens grundlegende Aspekte informatischer Bildung erweiterte Medienkompetenz ist nötige Voraussetzung für die kritische Rezeption und verantwortungsvolle Partizipation in der nächsten Gesellschaft (vgl. Brinda et al. 2016; KMK 2016; Knaus 2016b, 114; Schelhowe 2016, 49-50; Knaus 2018; Tulodziecki 2018). Im Sinne der zuvor geforderten Grenzüberwindungen und Neuvernetzungen (vgl. Kapitel «Desiderata: Crossing Boundaries») sollten daher Medienbildung und informatische Bildung in geeigneter Form miteinander verbunden werden (vgl. u.a. Tulodziecki 2016, 18-21; Herzig 2016, 73 und 75-76; weiterführende Gedanken und Thesen in Tulodziecki 2017 und 2018). Exemplarisch möchte ich daher im Folgenden auf aktuelle Entwicklungen hinweisen, die diese ausstehende Neu-Vernetzung befördern und begleiten. 
KMK-Strategie zu «Bildung in der digitalen Welt»: Begriffe und Zuständigkeiten

Als prominentes und aktuelles Beispiel für eine solche Neu-Vernetzung kann die Strategie der Kultusministerkonferenz (KMK) zur «Bildung in der digitalen Welt» (KMK 2016) herangeführt werden, die am 8. Dezember 2016 in Berlin beschlossen wurde und deren Entwurf seitens der Fachgruppe Schule der GMK, der Initiative KBOM! und der Sektion Medienpädagogik der DGfE kritisch kommentiert wurde (vgl. u.a. GMK 2016; KBoM 2016). Unsere wesentlichen Kritikpunkte waren die Verwendung eines unklaren und funktionalistischen Bildungsbegriffs sowie die schwerlich nachvollziehbare Tatsache, dass die Erklärung hinter den Zielen des ausgefeilten Papiers zur Medienbildung in der Schule aus dem Jahr 2012 zurückblieb (vgl. Knaus 2017c).

Der Fokus des neuen Papiers wird auf «Digitalisierung» und «Lernen» gelegt, aber nicht auf Bildung, obwohl es der Titel nahelegen würde (vgl. GMK 2016); es fällt auch auf, dass darin eine klare Dominanz des Lernens mit Medien - also die mediendidaktische bzw. die anwendungsbezogene ${ }^{6}$ Perspektive - gegenüber dem Lernen über Medien oder deren aktiver Gestaltung vorherrscht. Beim Lesen entsteht daher stellenweise der Eindruck, dass sich in der mediendidaktischen Verwendung digitaler Medien zur Erreichung der definierten Kompetenzziele die pädagogische Leitkategorie Mündigkeit quasi «von selbst» einstellt - bedauerlicherweise ist das aber nicht ganz so einfach. Gerade aus bildungspolitischer Perspektive mag die Begriffskritik möglicherweise übertrieben akademisch anmuten, jedoch kann man in der öffentlichen Diskussion immer wieder erfahren, dass die Griffigkeiten der Hashtags zu Lasten von inhaltlichen Missverständnissen und Unklarheiten bei fachlichen Zuständigkeiten gehen. So sind beispielsweise die viel verwendeten Begriffskombinationen «digitale Welt» und «digitale Kompetenzen» oder auch «digitale Bildung» (vgl. Kapitel «Form: «Digitale Bildung» - Vom Hashtag zum Konzept») irreführend, da sie neue Schnittmengen und ungeklärte fachliche Zuständigkeiten erzeugen: Wer ist beispielsweise zu fragen, wenn ein Curriculum zu «digitalen Kompetenzen» entwickelt werden soll - Informatikerinnen und Informatiker oder Medienpädagoginnen und Medienpädagogen? Wissenschaftlich sind diese unklaren Grenzen aufgrund der damit verbundenen kreativitätsförderlichen interdisziplinären Schnittmengen und Neu-Vernetzungsmöglichkeiten zu begrüssen, im Sinne der bisherigen Organisation von Schule und der Ausbildung von Lehrenden in tradierter Fächerordnung bergen sie jedoch (übergangsweise) Schwierigkeiten (vgl. Knaus 2017c).

Im direkten Vergleich zum KMK-Papier 2012 zu Medienbildung in der Schule fehlen in der neuen Erklärung insbesondere die Handlungsfelder ausserschulische

6 Die Kritik am passiv-funktionalistisch Bildungsbegriff wurde nicht nur von erziehungswissenschaftlicher Seite vorgebracht: Seitens des Fachbereichs Informatik und Ausbildung/Didaktik der Informatik (IAD) der Gesellschaft für Informatik (GI) wurde kritisiert, dass «eine Beschränkung auf die Nutzung digitaler Medien in allen Unterrichtsfächern bedeuten [würde], die digitale Welt so zu akzeptieren, wie sie jetzt ist, und deren Weiterentwicklung anderen zu überlassen. Schule muss aber zu gesellschaftlicher Teilhabe befähigen» (Brinda 2016). 
Kooperationspartner, Schulentwicklung und die (technische) Unterstützung der Schulen (vgl. GMK 2016). Da der Umgang mit Medien stark ausserschulisch geprägt ist, sollten Schulen - gerade im Bereich der Medienerziehung und Medienbildung - mit Institutionen und Trägern Kultureller Bildung zusammenarbeiten bzw. zusammenarbeiten können (vgl. Niesyto 2004; Knaus 2018). Vereinzelt geschieht das bereits, aber klare Empfehlungen seitens der KMK würden die Zusammenarbeit in - für beide Seiten - sehr förderlicher Weise voranbringen (vgl. Kapitel «Desiderata: Crossing Boundaries»). Ein weiteres Handlungsfeld ist die Schulentwicklung, die sich vielerorts als der Hebel für Veränderungen von Schule erwiesen hat. Auch Fragen des (technischen) Supports fehlen in der aktuellen Erklärung. Die Leerstelle erklärt sich vermutlich mit der Zuständigkeit der Sachaufwandsträger, auf denen in den letzten Jahren aufgrund anderer Herausforderungen bereits enormer Kostendruck lastete. «Technischer Support» fungiert im Kontext von Medienbildungs- und Schulentwicklung als Chiffre für die generelle Unterstützung von Schulen: Wie einschlägige Studien immer wieder bestätigen, besteht ein positiver Zusammenhang zwischen der (technischen) Unterstützung von Schulen und den Häufigkeiten der unterrichtlichen Mediennutzung sowie deren inhaltliche Breite (vgl. u.a. Engel et al. 2014; Breiter et al. 2017).

Auch das im Papier vorgestellte Kompetenzmodell verfügt über Schwächen: Da geplant ist, es als Grundlage zukünftiger Weiterentwicklungen von Lehrplänen heranzuziehen, sollte die vorgelegte Kompilierung von Kompetenzen stärker systematisiert und theoretisch begründet werden (vgl. GMK 2016, 10 f.). Eine Schwäche des Kompetenzmodells zeigt sich beispielsweise darin, dass statt der Befähigung zur informationellen Selbstbestimmung der Begriff «Schützen» aufgeführt wird - was jedoch keine Kompetenz ist. In Anbetracht der Komplexität, die mit der Entwicklung von Bildungsplänen und Kompetenzmodellen verbunden ist, und da die Entwicklungen in den Ländern vereinzelt schon weiter fortgeschritten sind (vgl. hierzu u.a. die LKM-Konzepte aus 2008 und 2015, www.laenderkonferenz-medienbildung.de), wäre es wahrscheinlich zielführender und anknüpfungsfähiger statt eines Modells lediglich einen Kompetenzrahmen anzubieten (vgl. GMK 2016, 9).

Während das KMK-Papier zu Medienbildung in der Schule nahezu resonanzlos blieb (vgl. u.a. Sulewski 2016; Knaus/Engel 2016), erzeugte das neue Papier - trotz aller Kritik - aufgrund des klaren Bekenntnisses der KMK ein grosses öffentliches und fachliches Interesse. Dies zeigt, dass wesentliche Themen nur dann wirklich vorankommen, wenn die KMK sie aufgreift und damit Handlungssicherheit für die beteiligten Akteurinnen und Akteure auf allen Ebenen schafft (vgl. Knaus 2017c).

Was an der neuen KMK-Erklärung ebenfalls als sehr positiv bewertet werden kann, ist die Tatsache, dass erstmals eine Erklärung der KMK zur Vorab-Kommentierung veröffentlicht wurde. Diese neue Kommentierungs- und Partizipationsstrategie sorgte für eine intensive Auseinandersetzung und mündete in konkrete 
Verbesserungsempfehlungen. Die Erklärung behandelt also nicht nur im gegenständlichen Sinne die Herausforderungen, die digitale Technik an die Gesellschaft und das Bildungssystem stellt, sondern die KMK nutzte mit dem vorherigen Einbezug der Fachgesellschaften auch das Potential, das digitale Medien zur Kommunikation, Kollaboration und Partizipation bieten. Dies erscheint mir in Anbetracht vieler weiterer komplexer Aufgaben, die eine fachübergreifende Expertise, überregionale Beteiligung und Kooperation erfordern, als ein kluger und weiterhin verfolgungswürdiger Weg.

\section{Dagstuhl-Erklärung: Aufeinanderzubewegen}

Als erster Organisationsversuch des zuvor geschilderten Schnittstellenproblems kann die Dagstuhl-Erklärung 2016 aufgefasst werden, die von Informatikerinnen und Informatikern sowie Medienpädagoginnen und Medienpädagogen gemeinsam verfasst wurde (vgl. Brinda et al. 2016) und damit eine bereits als historisch bezeichnete Wende einläutete (vgl. Schelhowe 2016, 43). Zwar wurde von beiden Seiten das Verfassen des Papiers als herausfordernd beschrieben und es wird auch nach seiner Veröffentlichung weiter kritisiert und diskutiert - das muss so sein, denn Kritik ist ein wesentliches Kennzeichen von Wissenschaft - aber gut ist, dass ein erster Schritt der Neu-Vernetzung unternommen wurde. Im Juli 2017 wurde der gemeinsame Austausch zwischen Informatik und Medienpädagogik um die Medienwissenschaft erweitert und im Rahmen der Klausurtagung zum «Verhältnis von Medienbildung und informatischer Bildung: Dagstuhl-Dreieck in progress» (KBoM 2017) im FTzM in Frankfurt am Main fortgesetzt.

\section{Aktuelle Calls: Neue Perspektiven, neue Rollenbilder und neue Begegnungen}

Vor wenigen Jahren hätte die Empfehlung, Medienbildung und informatische Bildung gemeinsam zu denken, möglicherweise hohe Wellen innerhalb der Communities geschlagen (vgl. u.a. Herzig 2016, 60). Wahrscheinlich liest sich mein Aufruf zur NeuVernetzung und praktischen Zusammenarbeit zwischen technisch-gestalterischen und pädagogischen Disziplinen bereits in naher Zukunft schon nicht mehr als «Häresie»? Zu wünschen wäre es - zumindest lassen dies die neueren Publikationen und aktuellen Calls des wohl wichtigsten publizistischen Hubs der Community vermuten: Das von Klaus Rummler, Beat Döbeli Honegger, Heinz Moser und Horst Niesyto herausgegebene Heft 25 der Onlinezeitschrift MedienPädagogik wurde mit «Medienbildung und informatische Bildung - quo vadis?» betitelt. Heidi Schelhowe, Gerhard Tulodziecki, Benjamin Jörissen und Bardo Herzig reflektieren darin die «kulturellen Möglichkeitsbedingungen» des Digitalen, analysieren Berührungspunkte zwischen Medienbildung und informatischer Bildung und geben konkrete Empfehlungen 
wechselseitiger Integrationsoptionen. Das geplante Themenheft der merz zu Medienpädagogik und Informatik von Kathrin Demmler und Björn Maurer (4/2018) und der aktuelle Call zur Medienpädagogik und Didaktik der Informatik von Torsten Brinda, Ira Diethelm, Sven Kommer und Klaus Rummler zeigen, dass sich eines der genannten Desiderate bereits in der Umsetzung befindet. Es passiert also einiges, was sich zu beobachten und zu begleiten lohnt - mein Beitrag dazu soll vorerst mit einer Empfehlung für ein neues Rollenbild der Medienpädagogik schliessen.

\section{Vision: Von der Torwächterin zur Lokomotivführerin}

Im Verlauf der vorliegenden Ausführungen sollte die anfänglich aufgestellte These belegt werden, dass die Medienpädagogik den aktuellen technologischen, technischen und gesellschaftlichen Entwicklungen in einem Verständnis als «Torwächterin», die primär vor "schädlichen» Medien schützen möchte, nicht begegnen kann (vgl. Kapitel «Turn: Vom Medienschutz zur Medienpädagogik»).

Doch welche Rolle sollte die Medienpädagogik stattdessen in der nächsten Gesellschaft innehaben? Wie wäre es mit der Rolle der Lokomotivführerin? Immerhin war die Lokomotive stets eine Metapher für (technischen) Fortschritt. Zwar ist diese Metapher inzwischen etwas aus der Zeit gefallen, dennoch verfügt das Bild der Lokomotivführerin über anknüpfbare Analogien für die Rolle der Medienpädagogik in der nächsten Gesellschaft wie Dirk Baecker (2007) sie beschreibt: Eine Lokomotivführerin schaut (1) nach vorn, sie antizipiert künftige Entwicklungen, denkt mit und denkt voraus. Eine Lokomotivführerin übernimmt (2) auch Verantwortung, sie denkt dabei stets an die individuellen und gesellschaftlichen Folgen von (Bildungs-)Entscheidungen. Sie braucht dafür (3) ein gutes Team im Zug: also gut ausgebildete Praktikerinnen und Praktiker - echte Profis eben; und sie benötigt (4) auch gute Kontakte ausserhalb des Zuges, wie beispielsweise «Funkkontakt» zu anderen Lokführerinnen und Lokführern zum Erfahrungsaustausch. Nicht zuletzt benötigt sie (5) eine gute Verbindung zur «Leitstelle», um wichtige Weichenstellungen in Gesellschaft und Politik zu beeinflussen. Und, da (6) ein Zug nicht zurückfahren kann, muss eine Lokführerin sehr gut wissen, wann sie bremsen muss. Daher komme auch ich hier zum Punkt.

\section{Literatur}

Aßmann, Sandra, Niels Brüggen, Valentin Dander, Harald Gapski, Gerda Sieben, Angela Tillmann, und Isabel Zorn. 2016. «Digitale Datenerhebung und -verwertung als Herausforderung für Medienbildung und Gesellschaft - Ein medienpädagogisches Diskussionspapier zu Big Data und Data Analytics». In Kommunikationskulturen in digitalen Welten, hrsg. v. Marion Brüggemann, Thomas Knaus, und Dorothee Meister. München: kopaed, 131-139. 
Aufenanger, Stefan. 1997. «Medienpädagogik und Medienkompetenz - eine Bestandsaufnahme. In Medienkompetenz im Informationszeitalter, hrsg. v. d. Enquete-Kommission Zukunft der Medien in Wirtschaft und Gesellschaft. Bonn: Deutscher Bundestag, 15-22.

Baacke, Dieter. 1973. Kommunikation und Kompetenz. Grundlegung einer Didaktik der Kommunikation und ihrer Medien. München: Juventa.

Baacke, Dieter. 1996: Medienpädagogik. Grundlagen der Medienkommunikation. Bd. 1. Tübingen: Niemeyer.

Baecker, Dirk. 2007. Studien zur nächsten Gesellschaft. Frankfurt am Main: Suhrkamp.

Beer, Raphael. 2007. Erkenntniskritische Sozialisationstheorie - Kritik der sozialisierten Vernunft. Wiesbaden: VS.

Bitkom. 2015. Digitale Schule und vernetztes Lernen. http://www.bitkom.org/Bitkom/Publikationen/Digitale-Schule-und-vernetztes-Lernen.html.

Blumer, Herbert. 1969. Symbolic Interactionism: Perspective and Method. New Jersey (USA): Prentice-Hall.

Bos, Wilfried, Birgit Eickelmann, Julia Gerick, Frank Goldhammer, Heike Schaumburg, Knut Schwippert, Martin Senkbeil, Renate Schulz-Zander, und Heike Wendt. 2014. ICILS 2013 Computer- und informationsbezogene Kompetenzen von Schülerinnen und Schülern in der 8. Jahrgangsstufe im internationalen Vergleich. Münster: Waxmann.

Breiter, Andreas, Anja Zeising, und Björn Eric Stolpmann. 2017. IT-Ausstattung an Schulen: Kommunen brauchen Unterstützung für milliardenschwere Daueraufgabe. http://www. bertelsmann-stiftung.de/de/publikationen/publikation/did/it-ausstattung-an-schulenkommunen-brauchen-unterstuetzung-fuer-milliardenschwere-daueraufgabe/.

Brinda, Torsten. 2016. Gl kritisiert reduzierten Bildungsbegriff der KMK: Informatik muss in Bildungsstrategie für die digitale Welt integriert werden (PM vom 25. Juli 2016). http://www. gi.de/aktuelles/meldungen/detailansicht/article/gi-kritisiert-reduzierten-bildungsbegriffder-kmk-informatik-muss-in-bildungsstrategie-fuer-die-dig.html.

Brinda, Torsten, Ira Diethelm, Rainer Gemulla, Ralf Romeike, Johannes Schöning, Carsten Schulte, und et al. 2016. "Dagstuhl-Erklärung: Bildung in der digitalen vernetzten Welt». https://www.gi.de/aktuelles/meldungen/detailansicht/article/dagstuhl-erklaerung-bildung-in-der-digitalen-vernetzten-welt.html.

Brüggen, Niels, Guido Bröckling, und Ulrike Wagner. 2017. Bildungspartnerschaften zwischen Schule und außerschulischen Akteuren der Medienbildung, herausgegeben von FSM - Freiwillige Selbstkontrolle Multimedia-Dienstanbieter e.V. Berlin. http://www.medien-in-dieschule.de/bildungspartnerschaften.

Calmbach, Marc, Silke Borgstedt, Inga Borchard, Peter Martin Thomas, und Berthold Bodo Flaig. 2016. Wie ticken Jugendliche 2016? Lebenswelten von Jugendlichen im Alter von 14 bis 17 Jahren in Deutschland. Wiesbaden: Springer. https://doi.org/10.1007/978-3-658-12533-2.

Carstensen, Tanja, Christina Schachtner, Heidi Schelhowe, und Raphael Beer. 2014. «Subjektkonstruktionen im Kontext digitaler Medien». In Digitale Subjekte, hrsg. v. Tanja Carstensen, Christina Schachtner, Heidi Schelhowe, und Raphael Beer. Bielefeld: Transcript. 
Cooley, Charles Horton. 1972. Social Organization. A Study of the Larger Mind. New York: Schoken Books.

Deterding, Sebastian. 2011. «Was geht hier eigentlich vor sich? Medienrealität, Mediensozialisation und Medienkompetenz aus rahmenanalytischer Perspektive». In Medialität und Realität, hrsg. v. Johannes Fromme, Stefan Iske, und Winfried Marotzki, 103-126. Wiesbaden: VS Verlag für Sozialwissenschaften. https://doi.org/10.1007/978-3-531-92896-8_7.

DGfE Sektion Medienpädagogik. 2017. «Orientierungsrahmen für die Entwicklung von Curricula für medienpädagogische Studiengänge und Studienanteile». MedienPädagogik: Zeitschrift für Theorie und Praxis der Medienbildung, Dezember, 1-7. https://doi.org/10.21240/ mpaed/00/2017.12.04.X.

DIVSI. 2014. Kinder, Jugendliche und junge Erwachsene in der digitalen Welt (U25-Studie). http://www.divsi.de/publikationen/studien/divsi-u25-studie-kinder-jugendliche-und-junge-erwachsene-in-der-digitalen-welt/.

Drucker, Peter F. 2002. «The Next Society: A Survey of the Near Future». In Managing in the Next Society, hrsg. v. Peter Drucker, 233-299. New York: St. Martin's Press.

Engel, Olga, Thomas Knaus, und Katharina Thülen. 2014. Projekt fraLine (Abschlussbericht über die 4. Projektlaufzeit Sep. 2011 bis August 2014 und Rückblick über 12 Jahre Schul-ITService in Frankfurt am Main). http://ftzm.de/images/FTzM/Publikationen/Projektberichte/Abschlussbericht_fraLine4_2015.pdf.

Flusser, Vilém. 1998. Kommunikologie. Frankfurt am Main: Fischer.

Friedrich, Katja, Friederike Siller, und Albert Treber, Hrsg. 2015. Smart und mobil - Digitale Kommunikation als Herausforderung für Bildung, Pädagogik und Politik. München: kopaed.

Fritz, Karsten, Stephan Kersting, und Ralf Vollbrecht. 2003. Mediensozialisation - Pädagogische Perspektiven des Aufwachsens in Medienwelten. Wiesbaden: VS.

Ganguin, Sonja. 2004. «Medienkritik - Kernkompetenz unserer Mediengesellschaft». Ludwigsburger Beiträge zur Medienpädagogik 6: 1-7.

Gapski, Harald. 2015. Big Data und Medienbildung. Zwischen Kontrollverlust, Selbstverteidigung und Souveränität in der digitalen Welt, Schriftenreihe zur Digitalen Gesellschaft NRW, Bd. 3. München: kopaed.

Geulen, Dieter, und Klaus Hurrelmann. 1980. «Zur Programmatik einer umfassenden Sozialisationstheorie». In Handbuch Sozialisationsforschung, hrsg. v. Klaus Hurrelmann und Dieter Ulich, 51-67. Weinheim: Beltz.

GMK - Gesellschaft für Medienpädagogik und Kommunikationskultur. 2016. Stellungnahme der FG Schule der GMK zum Strategiepapier der Kultusministerkonferenz vom 12. Mai 2016 zu ‘Bildung in der digitalen Welt`. http://www.gmk-net.de/fileadmin/pdf/GMK-Stellungnahme_zum_KMK-Strategie-Entwurf.pdf.

Goethe, Johann Wolfgang von. 1999. Poetische Werke, Bd. 5. Essen: Phaidon Verlag.

Herzig, Bardo. 2016. «Medienbildung und Informatische Bildung - Interdisziplinäre Spurensuche». Hrsg. v. Klaus Rummler, Beat Döbeli Honegger, Heinz Moser, und Horst Niesyto. MedienPädagogik: Zeitschrift für Theorie und Praxis der Medienbildung 25 (Oktober): 59-79. https://doi.org/10.21240/mpaed/25/2016.10.28.X. 
Hoffmann, Bernward (2008): «Bewahrpädagogik». In Handbuch Medienpädagogik, hrsg. v. Uwe Sander, Friederike von Gross, und Kai-Uwe Hugger, 42-50. Wiesbaden: VS.

Hoffmann, Dagmar, Friedrich Krotz, und Wolfgang Reißmann. 2017. Mediatisierung und Mediensozialisation: Prozesse - Räume - Praktiken. Wiesbaden: VS.

Hoffmann, Dagmar, und Lothar Mikos. 2010. Mediensozialisationstheorien - Modelle und Ansätze in der Diskussion. Wiesbaden: VS.

Hurrelmann, Bettina. 2002. «Zur historischen und kulturellen Relativität des „gesellschaftlich handlungsfähigen Subjekts" als normative Rahmenidee für Medienkompetenz». In Medienkompetenz - Voraussetzungen, Dimensionen, Funktionen, hrsg. v. Norbert Groeben und Bettina Hurrelmann, 111-126. Weinheim: Juventa.

Hurrelmann, Klaus. 2006. Einführung in die Sozialisationstheorie. Weinheim: Beltz.

Hurrelmann, Klaus, Matthias Grundmann, und Sabine Walper. 2008. Handbuch Sozialisationsforschung. Weinheim: Beltz.

Hurrelmann, Klaus, Ullrich Bauer, Matthias Grundmann, und Sabine Walper. 2015. Handbuch Sozialisationsforschung. Weinheim: Beltz.

Iske Stefan. 2016. «Medienbildung im Kontext digitaler Personenprofile». In Von der Bildung zur Medienbildung. Medienbildung und Gesellschaft, hrsg. v. Dan Verständig, Jens Holze, und Ralf Biermann, 257-280. Wiesbaden: Springer VS.

Jellinek, Georg. 1914. Allgemeine Staatslehre. Berlin: O. Häring. http://archive.org/stream/allgemeinestaats00jelliala.

Jenkins, Henry. 2006. Convergence Culture. Where Old and New Media Collide. New York: New York University Press.

Joas, Hans. 1991. «Rollen- und Interaktionstheorien in der Sozialisationsforschung». In Neues Handbuch der Sozialisationsforschung, hrsg. v. Klaus Hurrelmann und Dieter Ulich, 137152. Weinheim: Beltz.

KBoM - Keine Bildung ohne Medien. 2016. Stellungnahme der Initiative Keine Bildung ohne Medien - KBoM! zum Strategiepapier der Kultusministerkonferenz vom 12. Mai 2016 zu ‘Bildung in der digitalen Welt. http://www.keine-bildung-ohne-medien.de/publications/stellungnahme-zum-kmk-strategiepapier-bildung-in-der-digitalen-welt/.

KBoM - Keine Bildung ohne Medien. 2017. Klausurtagung zum Verhältnis von informatischer Bildung und Medienbildung im Juli 2017 - Dagstuhl-Dreieck in progress (Tagungsankündigung). http://ftzm.de/medienbildung/klausurtag-zum-verhaeltnis-von-informatischerbildung-und-medienbildung.

Keil, Reinhard. 2006. «Zur Rolle interaktiver Medien in der Bildung». In Lernstätten im Wandel - Innovation und Alltag in der Bildung, hrsg. v. Reinhard Keil und Detlef Schubert, 59-77. Münster: Waxmann.

Keiner, Edwin. 2015. «Pädagogik, Erziehungswissenschaft, Bildungswissenschaft, Empirische Bildungsforschung - Begriffe und funktionale Kontexte». In Unscharfe Grenzen - eine Disziplin im Dialog: Pädagogik, Erziehungswissenschaft, Bildungswissenschaft, Empirische Bildungsforschung, hrsg. v. Edith Glaser, und Edwin Keiner, 13-34. Bad Heilbrunn: Klinkhardt. 
Keiner, Edwin. 2017. «Didaktik - Bildung - Technik - Kritik. Medienpädagogik und Antinomien der Moderne». Hrsg. v. Sven Kommer, Thorsten Junge, und Christiane Rust. MedienPädagogik: Zeitschrift für Theorie und Praxis der Medienbildung 27 (April): 270-86. https://doi. org/10.21240/mpaed/27/2017.04.29.X.

KMK - Kultusministerkonferenz. 2016. Bildung in der digitalen Welt. http://www.kmk.org/fileadmin/Dateien/pdf/PresseUndAktuelles/2016/Bildung_digitale_Welt_Webversion.pdf.

Knaus, Thomas, Dorothee M. Meister, und Gerhard Tulodziecki. 2017. «Futurelab Medienpädagogik: Qualitätsentwicklung - Professionalisierung - Standards». MedienPädagogik: Zeitschrift für Theorie und Praxis der Medienbildung, 1-23. https://doi.org/10.21240/ mpaed/00/2017.10.24.X.

Knaus, Thomas, und Olga Engel. 2015. «(Auch) auf das Werkzeug kommt es an - Technikhistorische und techniktheoretische Annäherungen an den Werkzeugbegriff in der Medienpädagogik». In fraMediale - digitale Medien in Bildungseinrichtungen (Bd. 4), hrsg. v. Thomas Knaus, und Olga Engel, 15-57. München: kopaed.

Knaus, Thomas, und Olga Engel, Hrsg. 2016. Wi(e)derstände - Digitaler Wandel in Bildungseinrichtungen. Bd. 5 der fraMediale-Reihe. München: kopaed.

Knaus, Thomas. 2009. Kommunigrafie. München: kopaed.

Knaus, Thomas. 2013. «Technik stört! Lernen mit digitalen Medien in interaktionistisch-konstruktivistischer Perspektive». In fraMediale - digitale Medien in Bildungseinrichtungen (Bd. 3), hrsg. v. Thomas Knaus, und Olga Engel, 21-60. München: kopaed.

Knaus, Thomas. 2015. «Me, my Tablet - and Us. Vom Mythos eines Motivationsgenerators zum vernetzten Lernwerkzeug für autonomopoietisches Lernen». In Smart und mobil - Digitale Kommunikation als Herausforderung für Bildung, Pädagogik und Politik, hrsg. v. Katja Friedrich, Friederike Siller, und Albert Treber, 17-42. München: kopaed.

Knaus, Thomas. 2016a. «Potentiale des Digitalen - Theoretisch-konzeptionelle Betrachtungen pädagogischer und didaktischer Potentiale des schulischen Einsatzes von Tablets und BYOD». In medien+erziehung: schule. smart. mobil 60: 33-39.

Knaus, Thomas. 2016b. «digital - medial - egal? - Ein fiktives Streitgespräch um digitale Bildung und omnipräsente Adjektive in der aktuellen Bildungsdebatte». In Kommunikationskulturen in digitalen Welten - Konzepte und Strategien der Medienpädagogik und Medienbildung, hrsg. v. Marion Brüggemann, Thomas Knaus, und Dorothee Meister, 99-130. München: kopaed.

Knaus, Thomas. 2016c. «Kooperatives Lernen. Begründungen - digitale Potentiale - konzeptionelle Perspektiven. In Perspektiven für die digitale Weiterbildung - Bildungslandschaften der Zukunft, hrsg. v. August Wilhelm Scheer, und Christian Wachter, 141-155. Saarbrücken: IMC AG.

Knaus, Thomas. 2017a. «Pädagogik des Digitalen - Phänomene - Potentiale - Perspektiven». In Software takes command, hrsg. v. Sabine Eder, Claudia Mikat, und Angela Tillmann. München: kopaed, 49-68. 
Knaus, Thomas. 2017b. «Verstehen - Vernetzen - Verantworten. Warum Medienbildung und informatische Bildung uns alle angehen und wir sie gemeinsam weiterentwickeln sollten». In Informatische Bildung zum Verstehen und Gestalten der digitalen Welt, hrsg. v. Ira Diethelm, LNI P-274: 31-48. Bonn: GI.

Knaus, Thomas. 2017c. «Bildung in der digitalen Welt - Das Strategiepapier der KMK in der Diskussion». Redemanuskript des Impulsvortrags im Rahmen der interdisziplinären Fachtagung Digitale Welt als Thema in Schule und Unterricht am 15.11.2017 der Bundeszentrale für politische Bildung (bpb) und der Kultusministerkonferenz (KMK) in Berlin.

Knaus, Thomas. 2018. «Technikkritik und Selbstverantwortung - Plädoyer für ein erweitertes Medienkritikverständnis». In Medienkritik im digitalen Zeitalter, hrsg. v. Horst Niesyto, und Heinz Moser. München: kopaed [im Erscheinen].

Kommer, Sven. 2016. «Buch statt Tablet-PC. Warum die digitalen Medien nicht in die Schule kommen - der Faktor LehrerIn». In Wi(e)derstände - Digitaler Wandel in Bildungseinrichtungen (Bd. 5 der fraMediale-Reihe), hrsg. v. Thomas Knaus und Olga Engel, 35-46. München: kopaed.

Krotz, Friedrich. 2016. "Wandel von sozialen Beziehungen, Kommunikationskultur und Medienpädagogik - Thesen aus der Perspektive des Mediatisierungsansatzes». In Kommunikationskulturen in digitalen Welten, hrsg. v. Marion Brüggemann, Thomas Knaus, und Dorothee M. Meister, 19-42. München: kopaed.

Lange, Andreas. 2015. "Sozialisation in der mediatisierten Gesellschaft». In Handbuch Sozialisationsforschung, hrsg. v. Klaus Hurrelmann, Ullrich Bauer, Matthias Grundmann, und Sabine Walper, 537-556. Weinheim: Beltz.

Latour, Bruno. 1996. Der Berliner Schlüssel. Erkundungen eines Liebhabers der Wissenschaften (La clef de Berlin et autres leçons d'un amateur de sciences). Berlin: Akademie-Verlag.

Lorenz, Ramona, und Wilfried Bos. 2015. «Konzeption, Anlage und Durchführung des Projekts Schule digital - der Länderindikator 2015». In Schule digital - der Länderindikator 2015, hrsg. v. Wilfried Bos, Ramona Lorenz, Manuela Endberg, Heike Schaumburg, Renate Schulz-Zander, und Martin Senkbeil. Münster: Waxmann.

Manovich, Lev. 2001. The Language of New Media. Cambridge: MIT Press.

Mayrberger, Kerstin, Johannes Fromme, Petra Grell, und Theo Hug, Hrsg. 2017. Jahrbuch Medienpädagogik 13. Vernetzt und Entgrenzt - Gestaltung von Lernumgebungen mit digitalen Medien. Wiesbaden: Springer VS. https://doi.org/10.1007/978-3-658-16432-4.

McLuhan, Marshall. 1968. Die Gutenberg Galaxis: Das Ende des Buchzeitalters. Düsseldorf: Econ. Mead, George Herbert. 1973. Geist, Identität und Gesellschaft. Frankfurt am Main: Suhrkamp.

Meder, Norbert. 1998. "Neue Technologien und Erziehung/Bildung». In Deutsche Gegenwartspädagogik, hrsg. v. Michele Borrelli, und Jörg Ruhloff, 3:26-40. Baltmannsweiler: Schneider Hohengehren.

Meusburger, Peter. 2009. "Räumliche Disparitäten des Wissens». In Komplexe Regionen, hrsg. v. Marissa Hey, und Kornelia Engert, 209-229. Wiesbaden: VS Verlag für Sozialwissenschaften. https://doi.org/10.1007/978-3-531-91619-4.

Meyer-Drawe, Käte. 1990. Illusionen von Autonomie. München: P. Kirchheim. 
Moser, Heinz. 2010a. Einführung in die Medienpädagogik. Wiesbaden: VS.

Moser, Heinz. 2010b. «Digital Divide in den Zeiten von Web 2.0 und Social Networks». In Fokus Medienpädagogik. Aktuelle Forschungs- und Handlungsfelder, hrsg. v. Petra Bauer, Hannah Hoffmann, und Kerstin Mayrberger, 135-149. München: kopaed.

MPFS - Medienpädagogischer Forschungsverbund Südwest. 2014. KIM 2014 - Kinder + Medien, Computer + Internet. Stuttgart: MPFS.

MPFS - Medienpädagogischer Forschungsverbund Südwest. 2016. JIM 2016 - Jugend, Information, (Multi-)Media. Stuttgart: MPFS.

Nake, Frieder. 1992. "Informatik und die Maschinisierung von Kopfarbeit». In Sichtweisen der Informatik, hrsg. v. Wolfgang Coy, Frieder Nake, Jörg-Martin Pflüger, Arno Rolf, Jürgen Seetzen, Dirk Siefkes, und Reinhard Stransfeld, 181-201. Braunschweig: Vieweg.

Niesyto, Horst. 2004. «Öffnung von Schule und partnerschaftliche Kooperation. Zur Zusammenarbeit von schulischer und außerschulischer Medienarbeit». In Medienbildung im Doppelpack. Wie Schule und Jugendhilfe einander ergänzen können, hrsg. v. Ida Pöttinger, Wolfgang Schill, und Günter Thiele, 39-49. München: kopaed.

Niesyto, Horst. 2006. «Medienkritik und Mediensozialisation». In Medienkritik Heute: Grundlagen, Beispiele und Praxisfelder, hrsg. v. Horst Niesyto, Matthias Rath, und Hubert Sowa, 53-70. München: kopaed.

Niesyto, Horst. 2008. «Medienkritik». In Handbuch Medienpädagogik, hrsg. v. Uwe Sander, Friederike von Gross, und Kai-Uwe Hugger, 129-135. Wiesbaden: VS.

Niesyto, Horst. 2012. «Medienpädagogik in der Lehrerbildung in Baden-Württemberg. Konzeptionelle Überlegungen und praktische Schritte zu einer medienpädagogischen Grundbildung». In Jahrbuch Medienpädagogik 9, hrsg. v. Renate Schulz-Zander, Birgit Eickelmann, Heinz Moser, Horst Niesyto, und Petra Grell, 333-357. Wiesbaden: VS Verlag für Sozialwissenschaften. https://doi.org/10.1007/978-3-531-94219-3_15.

Niesyto, Horst. 2014. «Grundbildung Medien an der Pädagogischen Hochschule Ludwigsburg». In Grundbildung Medien in pädagogischen Studiengängen, hrsg. v. Peter Imort, und Horst Niesyto, 125-138. München: kopaed.

Niesyto, Horst. 2016. «Keine Bildung ohne Medien! - Kritische Medienbildung jenseits funktioneller Vereinnahmung». In Wi(e)derstände - Digitaler Wandel in Bildungseinrichtungen (Bd. 5 der fraMediale-Reihe), hrsg. v. Thomas Knaus, und Olga Engel, 17-34. München: kopaed.

Nohl, Arnd-Michael. 2011. Pädagogik der Dinge. Bad Heilbrunn: Klinkhardt.

Pietraß, Manuela. 2004. «Medienkompetenz als „Framing“. Grundlagen einer rahmenanalytischen Bestimmung von Medienkompetenz». In Medienkompetenz und Medienleistungen in der Informationsgesellschaft, hrsg. v. Heinz Bonfadelli, Priska Bucher, Ingrid Paus-Hasebrink, und Daniel Süss, 10-21. Zürich: Pestalozzianum.

Puentedura, Ruben R. 2014. Learning, Technology, and the SAMR Model: Goals, Processes, and Practice. http://www.hippasus.com/rrpweblog/archives/000127.html.

Schachtner, Christina, und Nicole Duller. 2014. «Kommunikationsort Internet». In Digitale Subjekte, hrsg. v. Tanja Carstensen, Christina Schachtner, Heidi Schelhowe, und Raphael Beer. Bielefeld: Transcript. 
Schelhowe, Heidi. 1997. Das Medium aus der Maschine. Zur Metamorphose des Computers. Frankfurt am Main: Campus.

Schelhowe, Heidi. 2007. Technologie, Imagination und Lernen - Grundlagen für Bildungsprozesse mit Digitalen Medien. Münster: Waxmann.

Schelhowe, Heidi. 2016. «Through the Interface» - Medienbildung in der digitalisierten Kultur». Hrsg. v. Klaus Rummler, Beat Döbeli Honegger, Heinz Moser, und Horst Niesyto. MedienPädagogik: Zeitschrift für Theorie und Praxis der Medienbildung 25 (Oktober): 41-58. https://doi.org/10.21240/mpaed/25/2016.10.27.X.

Schorb, Bernd. 1995. Medienalltag und Handeln. Medienpädagogik in Geschichte, Forschung und Praxis. Opladen: Leske+Budrich.

Schorb, Bernd. 2005. «Sozialisation». In Grundbegriffe Medienpädagogik, hrsg. v. Jürgen Hüther, und Bernd Schorb, 381-388. München: kopaed.

Seel, Norbert M., und Ulrike Hanke. 2015. Erziehungswissenschaft. Wiesbaden: VS.

Spanhel, Dieter. 2013. «Sozialisation in mediatisierten Lebenswelten - Grundzüge eines theoretischen Bezugsrahmens». merz-Wissenschaft 57 (6): 30-43.

Stiller, Michael, und Hannah Bolz. 2016. «Die Verankerung der Medienpädagogik in der universitären Lehrendenbildung - Der Auf- und Ausbau des Erweiterungsstudiengangs Medienpädagogik». In Wi(e)derstände - Digitaler Wandel in Bildungseinrichtungen (Bd. 5 der fraMediale-Reihe), hrsg. v. Thomas Knaus, und Olga Engel, 123-138. München: kopaed.

Sulewski, Horst. 2016. «Konzept gelungen - und die Praxis? Entwicklungen der schulischen Medienbildung nach der KMK-Erklärung von 2012». In Wi(e)derstände - Digitaler Wandel in Bildungseinrichtungen (Bd. 5 der fraMediale-Reihe), hrsg. v. Thomas Knaus, und Olga Engel, 69-84. München: kopaed.

Süss, Daniel, Claudia Lampert, und Christine W. Wijnen. 2013. Medienpädagogik. Wiesbaden: VS.

Sutter, Tilmann. 1999. Systeme und Subjektstrukturen. Zur Konstitutionstheorie des interaktionistischen Konstruktivismus. Wiesbaden: VS.

Swertz, Christian. 2007: Bildungstechnologische Medienpädagogik. In Handbuch Medienpädagogik hrsg. v. Uwe Sander, Friederike von Gross, und Kai Hugger, 66-74, Wiesbaden: VS.

Thye, Iris. 2013. Kommunikation und Gesellschaft - systemtheoretisch beobachtet. Sprache, Schrift, einseitige Massen- und digitale Online-Medien. Wiesbaden: VS.

Tillmann, Klaus-Jürgen. 2010, Sozialisationstheorien. Eine Einführung in den Zusammenhang von Gesellschaft, Institution und Subjektwerdung. Reinbek: Rowohlt.

Tulodziecki, Gerhard. 1997. Medien in Erziehung und Bildung. Grundlagen und Beispiele einer handlungs- und entwicklungsorientierten Medienpädagogik. Bad Heilbrunn: Klinkhardt.

Tulodziecki, Gerhard. 2012. «Medienpädagogische Kompetenz und Standards in der Lehrerbildung». In Jahrbuch Medienpädagogik 9, hrsg. v. Renate Schulz-Zander, Birgit Eickelmann, Heinz Moser, Horst Niesyto, und Petra Grell, 271-397. Wiesbaden: VS Verlag für Sozialwissenschaften. https://doi.org/10.1007/978-3-531-94219-3. 
Tulodziecki, Gerhard. 2016. «Konkurrenz oder Kooperation? Zur Entwicklung des Verhältnisses von Medienbildung und informatischer Bildung». Herausgegeben von Klaus Rummler, Beat Döbeli Honegger, Heinz Moser, und Horst Niesyto. MedienPädagogik: Zeitschrift für Theorie und Praxis der Medienbildung 25 (Oktober): 7-25. https://doi.org/10.21240/ mpaed/25/2016.10.25.X.

Tulodziecki, Gerhard. 2017. «Thesen zu einem Curriculum zur Bildung in einer durch Digitalisierung und Mediatisierung beeinflussten Welt». In merz, 61. Jhrg., Nr. 2 (April 2017), 50-56.

Tulodziecki, Gerhard. 2018. «Medienbildung angesichts von Digitalisierung und Mediatisierung». In Spannung? Potentiale! Spannungsfelder und Bildungspotentiale des Digitalen (Bd. 6 der fraMediale-Reihe), hrsg. v. Thomas Knaus, und Olga Engel. München: kopaed [im Erscheinen].

Tulodziecki, Gerhard, Bardo Herzig, und Silke Grafe. 2010. Medienbildung in Schule und Unterricht. Bad Heilbrunn: Klinkhardt.

Vollbrecht, Ralf, und Claudia Wegener. 2010. Handbuch Mediensozialisation. Wiesbaden: VS.

Vollbrecht, Ralf. 2001. Einführung in die Medienpädagogik, Weinheim: Beltz.

Wagner, Ulrike, und Sebastian Ring. 2016. «Organisierte Wildnis - Kooperation von außerschulischer und schulischer Medienpädagogik». In Wi(e)derstände - Digitaler Wandel in Bildungseinrichtungen (Bd. 5 der fraMediale-Reihe), hrsg. v. Thomas Knaus, und Olga Engel, 139-149. München: kopaed.

Wenger, Etienne. 1998. Communities of Practice: Learning, Meaning, and Identity. Cambridge (UK): Cambridge University Press.

Wing, Jeanette M. 2006. «Computational Thinking». Communications of the ACM, 49, 3: 33-35.

\section{Ah jetzt ja ... noch eine Insel mit zwei Bergen?}

Wie jede Metapher hat natürlich auch die vorgeschlagene Analogie der Lokomotivführerin gewisse Grenzen: So determinieren die Schienen, die ein Zug nicht verlassen kann, die freien Entscheidungen der Lokführerin. Für die Medienpädagogik einer nächsten Gesellschaft wünscht man sich jedoch umfangreichere Freiräume und Gestaltungsmöglichkeiten.

In Anbetracht der gewählten Metapher, die offenbar an dieser Stelle an ihre Grenzen stößt, fällt mir ein noch krasserer Determinismus ein: Ich denke an Lukas, den Lokomotivführer, aus der Augsburger Puppenkiste, der auf Lummerland - der «Insel mit den zwei Bergen [...] mit viel Tunnels und Geleisen» - stets nur im Kreis fuhr! Aber wie war das noch gleich in Jim Knopf und die Wilde 13? In dieser Geschichte musste sogar Lukas sein Lummerland verlassen: Er dichtete seine Lokomotive ab und fuhr mit ihr hinaus aufs blaue Meer. 